\title{
Cavity Photons as a Probe for Charge Relaxation Resistance and Photon Emission in a Quantum Dot Coupled to Normal and Superconducting Continua
}

\author{
L. E. Bruhat, ${ }^{1}$ J. J. Viennot, ${ }^{1,2}$ M. C. Dartiailh, ${ }^{1}$ M. M. Desjardins, ${ }^{1}$ T. Kontos, ${ }^{1}$ and A. Cottet ${ }^{1, *}$ \\ ${ }^{1}$ Laboratoire Pierre Aigrain, Ecole Normale Supérieure-PSL Research University, CNRS, \\ Université Pierre et Marie Curie-Sorbonne Universités, Université Paris Diderot-Sorbonne Paris Cité, \\ 24 rue Lhomond, F-75231 Paris Cedex 05, France \\ ${ }^{2}$ JILA and Department of Physics, University of Colorado, Boulder, Colorado 80309, USA \\ (Received 11 November 2015; revised manuscript received 4 March 2016; published 9 May 2016)
}

\begin{abstract}
Microwave cavities have been widely used to investigate the behavior of closed few-level systems. Here, we show that they also represent a powerful probe for the dynamics of charge transfer between a discrete electronic level and fermionic continua. We have combined experiment and theory for a carbon nanotube quantum dot coupled to normal metal and superconducting contacts. In equilibrium conditions, where our device behaves as an effective quantum dot-normal metal junction, we approach a universal photon dissipation regime governed by a quantum charge relaxation effect. We observe how photon dissipation is modified when the dot admittance turns from capacitive to inductive. When the fermionic reservoirs are voltage biased, the dot can even cause photon emission due to inelastic tunneling to/from a BardeenCooper-Schrieffer peak in the density of states of the superconducting contact. We can model these numerous effects quantitatively in terms of the charge susceptibility of the quantum dot circuit. This validates an approach that could be used to study a wide class of mesoscopic QED devices.
\end{abstract}

DOI: 10.1103/PhysRevX.6.021014

\section{INTRODUCTION}

Circuit QED techniques [1] have recently been put forward to investigate the electronic dynamics in quantum dot circuits [2-4] or, more generally, mesoscopic circuits [5]. So far, the interaction between cavity photons and charges [6-16] or spins [17] confined in quantum dots has received the most experimental attention. This atomiclike limit is a priori very appealing for quantum information applications since it goes, in principle, with long coherence times. Nevertheless, mesoscopic circuits are inseparable from the existence of electronic reservoirs with Fermi seas. These fermionic reservoirs are not necessarily a drawback. For instance, a strong coupling between a dot and a normal metal enables one to revisit condensed matter problems such as the Kondo effect. Ferromagnetic contacts can be used to design spin quantum bits [17] or to study spindependent transport [18]. Superconducting contacts are crucial for the study of Cooper pair splitting $[19,20]$, Andreev bound states [21-23], and Majorana quasiparticles [24]. In principle, microwave cavities could represent a powerful tool to investigate these features [25-32].

\footnotetext{
*Corresponding author. cottet@1pa.ens.fr
}

Published by the American Physical Society under the terms of the Creative Commons Attribution 3.0 License. Further distribution of this work must maintain attribution to the author(s) and the published article's title, journal citation, and DOI.

\section{Subject Areas: Optics, Quantum Physics,} Semiconductor Physics
In this context, it is crucial to understand how tunneling processes between a discrete energy level and the continuum of states of a reservoir can affect cavity photons. This situation is epitomized by a single quantum dot circuit coupled to a cavity, a case that has been studied elusively so far $[3,8,33]$. A recent experiment has revealed that the quantum dot can add an effective capacitance or an inductance to the photon environment, depending on the transparency of its contacts [8]. However, the cavity dissipation expected, together with this effect, has been left unexplored. On the theory side, most experiments combining quantum dot circuits and microwave resonators have been interpreted by disregarding fermionic reservoirs or by using a Lindbladt equation suitable for dot-reservoir tunnel rates much smaller than the temperature of the experiment. An alternative approach is highly desirable for investigating the open contacts limit. Descriptions in terms of the charge susceptibility of the quantum dot circuits have recently been suggested [5,34-36].

In this work, we study experimentally and theoretically the behavior of a single quantum dot in a carbon nanotube, coupled to normal metal $(\mathrm{N})$ and superconducting $(\mathrm{S})$ reservoirs, and embedded in a high finesse microwave cavity. In a first step, we study a dot with a discrete level coupled only to the $\mathrm{N}$ reservoir. In this case, the current response of the dot to a direct gate voltage excitation $V_{\text {rf }}$ can be developed as $I_{\mathrm{rf}}=i \omega_{\mathrm{rf}} C_{Q}\left(1-i \omega_{\mathrm{rf}} R_{\mathrm{ac}} C_{Q}\right) V_{\mathrm{rf}}+$ $o\left(\omega_{\mathrm{rf}}^{2}\right)$, provided the frequency $\omega_{\mathrm{rf}}$ of the excitation is smaller than the tunnel rate $\Gamma_{N}$ of the $\mathrm{N}$-dot junction. 
This development maps onto that expected for a RC circuit with capacitance $C_{Q}$ and resistance $R_{\mathrm{ac}}$. The capacitance $C_{Q}$ characterizes the ability of the dot to host electrons at dc or low frequencies. The resistance $R_{\mathrm{ac}}$ describes the dynamics of charge tunneling through the junction, which leads to the relaxation of the charge imbalance caused by $V_{\mathrm{rf}}$. For an incoherent device, $R_{\mathrm{ac}}$ depends on the detailed properties of the dot circuit. However, in the coherent noninteracting limit with $\omega_{\mathrm{rf}} \ll \Gamma_{N}$, Büttiker and coworkers have predicted that $R_{\mathrm{ac}}$ should take a universal value, independently of the dot parameters [37,38]. This effect, which reveals the wavy behavior of electrons inside the dot, is of fundamental as well as practical importance in the context of the miniaturization of electronic devices. The only observation of a universal $R_{\mathrm{ac}}$ so far was performed by Gabelli et al. with a strongly spin-polarized GaAs quantum dot, using ac conductance measurements [39]. Remarkably, this dot was in a noninteracting regime because of a top gate with an unusually large capacitance [40]. Noticeably, the independence of $R_{\mathrm{ac}}$ from the dot orbital energy was not tested by Gabelli and coworkers. In our system, this property appears as a scaling between the dissipation and dispersion induced by the dot on the cavity. We can experimentally confirm this scaling behavior for intermediate tunnel rates $\Gamma_{N} \sim 2.5 \omega_{\mathrm{rf}}$, where it is already approximately valid and well resolvable. Remarkably, this effect occurs in spite of the presence of strong Coulomb blockade in our sample. The effect of interactions on quantum charge relaxation has caused intense theoretical activity because, in practice, most quantum dots are subject to strong Coulomb interactions. In this limit, predictions for $R_{\mathrm{ac}}$ display a rich phenomenology [41-47]. Nevertheless, it was recently suggested that a universal charge relaxation resistance persists in the spin-degenerate interacting case $[45,46]$. Our results are consistent with this prediction. Finally, we observe how quantum charge relaxation depends on the dot orbital energy for smaller tunnel rates, when the dot admittance turns from capacitive to inductive.

In a second step, we study the finite bias voltage regime where the dot level is also coupled to the $\mathrm{S}$ reservoir. Contextually, the implementation of mesoscopic QED experiments with superconducting hybrid circuits is very recent. Atomic contacts between superconductors have been used to form a new type of quantum bit based on Andreev bound states [23]. Semiconducting nanowires have been used for realizing Josephson junctions in superconducting circuits $[48,49]$. However, quantum dot circuits with superconducting reservoirs have been coupled neither to microwave cavities nor to a direct ac excitation, so far. Despite this lack of experiments, photon-assisted tunneling between a dot and a superconductor has created theoretical interest for more than 15 years [50-59]. The coupling between superconductor-quantum dot hybrid circuits and microwave cavities has also been studied in recent theoretical works [25-27]. Here, we show experimentally that a microwave cavity is able to reveal photon-assisted tunnel events between a dot and the Bardeen-Cooper-Schrieffer (BCS) peaks of a superconductor, not visible in the dot current. In particular, we observe negative photon damping, which reveals photon emission. This result illustrates that quasiparticle tunneling to a fermionic reservoir does not always induce photonic dissipation.

In order to understand our measurements, we use a Keldysh Green's function approach. We can simultaneously reproduce the quantum dot conductance and the microwave response of the cavity, versus the dot gate and bias voltages, with an unprecedented accuracy for this type of hybrid system. We thereby validate the description of mesoscopic QED experiments in terms of an electronic charge susceptibility. To illustrate the broad scope of this approach, we present its multidot generalization, which can be used for many different geometries, for instance, Cooper pair splitters and topological hybrid nanocircuits. Our work also gives wide experimental perspectives since microwave cavities appear as a powerful probe for quantum charge relaxation, photon-assisted tunneling, and all other effects involving tunneling between a discrete level and fermionic reservoirs.

This article is organized as follows. Section II presents our experimental setup. Sections III and IV analyze our experimental data, for the $\mathrm{N}$-dot and $\mathrm{N}$-dot-S limits, respectively. Section V summarizes our results, presents the multidot generalization of our approach, and gives various perspectives. Appendix A shows experimental details and supplementary data. Appendix B discusses our theoretical approach.

\section{EXPERIMENTAL SETUP}

We use a carbon nanotube on which we evaporate a superconducting contact (S) surrounded by two normal metal $(\mathrm{N})$ contacts, visible in Fig. 1(b) (technical details are presented in Appendix A). In our regime of parameters, the whole nanotube section between the two $\mathrm{N}$ contacts forms a single quantum dot. An effective gate voltage $V_{g}$ is used to tune the dot-level orbital energy $\varepsilon_{d}$. We connect the $\mathrm{S}$ contact to ground, and we apply the same bias voltage $V_{b}$ to the two $\mathrm{N}$ contacts, which can thus be considered as an effective single contact [60]. The dot is capacitively coupled to the central conductor of a superconducting coplanar waveguide cavity through a top gate TG [see Fig. 1(a)]. We measure the cavity transmission $b_{t} / b_{\text {in }}$ at a frequency $\omega_{\mathrm{rf}}$ equal to the bare cavity frequency $\omega_{0} \sim 2 \pi \times 6.65 \mathrm{GHz}$. We determine the phase shift $\Delta \varphi$ and the reduced amplitude shift $\Delta A / A_{0}$ of $b_{t} / b_{i n}$, which are caused by the presence of the quantum dot circuit, with $A_{0}$ the bare cavity transmission amplitude. Simultaneously, we measure the dc current $I$ and differential conductance $G$ through the dot. The current $I$ shows clear signatures of Coulomb blockade with a charging energy $E_{c} \approx 1.8 \mathrm{meV}$ [see Fig. 1(d)]. It also vanishes for a bias voltage $V_{b}$ smaller 


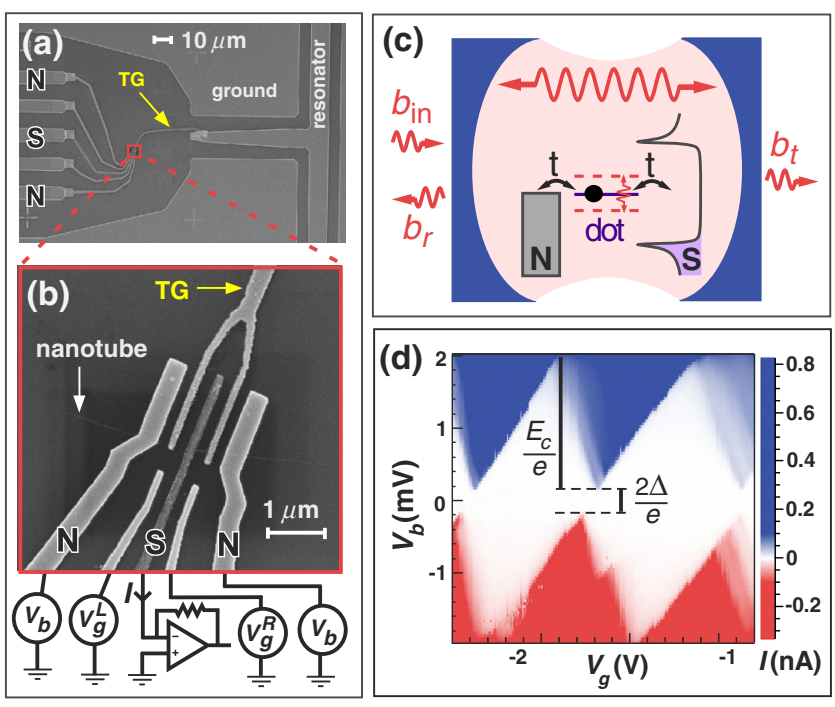

FIG. 1. Panels (a) and (b): Scanning electron micrograph of the microwave resonator and the quantum dot circuit. Panel (c): Principle of our setup. The dot level is tunnel coupled to the $\mathrm{N}$ and $\mathrm{S}$ reservoirs and modulated by the cavity electric field. Panel (d): Current through the $\mathrm{S}$ contact versus the effective gate voltage $V_{g}$ and the bias voltage $V_{b}$.

than the gap $\Delta \simeq 0.17 \mathrm{meV}$ of the $\mathrm{S}$ contact. Therefore, for $V_{b}=0$, the effect of the $\mathrm{S}$ contact can be disregarded, and the quantum dot circuit corresponds to an effective $\mathrm{N}$-dot junction, studied in Sec. III. For $e\left|V_{b}\right|>\Delta$, our device enables the study of quasiparticle transport in a N-dot-S bijunction, presented in Sec. IV.

\section{PHOTON DISSIPATION IN AN EFFECTIVE N-DOT JUNCTION}

A single dot level coupled to a $\mathrm{N}$ reservoir is the most basic configuration for studying the light matter interaction in a mesoscopic circuit. Our device realizes such a situation for $V_{b}=0$ due to the absence of subgap Andreev reflections. Figure 2 shows the cavity signals $\Delta \varphi$ (blue dots) and $\Delta A / A_{0}$ (red dots) versus the energy $\varepsilon_{d}$ of the dot orbital, for $V_{b}=0$, and decreasing tunnel rates $\Gamma_{N}$ from left to right and top to bottom panels. The correspondence between $\varepsilon_{d}$ and the gate voltage $V_{g}$ is given in Appendix A for each dot level. We observe resonances, although $I=0$. This means that the cavity is able to reveal quasiparticle tunneling between the dot and the $\mathrm{N}$ contact even if it does not lead to a dc current. The phase signal $\Delta \varphi$ (blue dots) can be positive as well as negative, as already observed in Ref. [8], depending on the value of $\Gamma_{N}$. This is because, for $\omega_{0} \ll \Gamma_{N}$, the quantum dot circuit behaves as an effective capacitance. Electrons can very rapidly follow the variations of the dot potential to go in and out of the dot, proportionally to the dot density of states. However, for $\omega_{0} \gg \Gamma_{N}$, the charge current lags behind the dot potential, so the dot behavior becomes inductive [37,61]. In contrast, the signal $\Delta A$ (red dots) always remains negative, up to experimental uncertainty. One could naively expect that $\Delta A$, which reveals photon dissipation, will scale with $\Gamma_{N}$, which is the main dissipation parameter in our problem. However, this intuition is wrong since $\Delta A$ becomes small when $\Gamma_{N}$ tends to large values [see Fig. 2(a)].

To understand the behavior of our device, we use the Hamiltonian

$$
\begin{aligned}
H_{\mathrm{tot}}= & H_{d}+\omega_{0} \hat{a}^{\dagger} \hat{a}+g\left(\hat{a}+\hat{a}^{\dagger}\right) \sum_{\sigma} \hat{d}_{\sigma}^{\dagger} \hat{d}_{\sigma} \\
& +\sum_{p} \omega_{p} \hat{b}_{p}^{\dagger} \hat{b}_{p}+\sum_{p}\left(\tau_{p} \hat{b}_{p}^{\dagger} \hat{a}+\tau_{p}^{*} \hat{a}^{\dagger} \hat{b}_{p}\right),
\end{aligned}
$$

where $H_{d}$ describes the quantum dot circuit (see Appendix B for details), $\hat{d}_{\sigma}^{\dagger}$ adds an electron with spin $\sigma$ in the dot level, $\hat{a}^{\dagger}$ adds a photon in the cavity, and $\hat{b}_{p}^{\dagger}$ describes a bosonic bath that accounts for the cavity intrinsic linewidth $\Lambda_{0}$. We assume that cavity photons modulate the chemical potential of the quantum dot with a coupling constant $g=e \varkappa V_{\text {rms }}$, with $V_{\text {rms }}$ the cavity root-mean-square voltage and $e$ the electron charge. The dimensionless coupling constant $\varkappa$ depends on the overlap between the electron wave function associated with the dot level and the photonic pseudopotential, which is spatially nonuniform [5]. Therefore, the value of $g$ generally depends on the dot level considered, as we will see in the experimental data. Using Eq. (1), a semiclassical linear response approach leads to the cavity transmission (see Appendix B):

$$
\frac{b_{t}}{b_{\text {in }}}=\frac{t_{0}}{\omega_{\mathrm{rf}}-\omega_{0}-i \Lambda_{0}-g^{2} \chi\left(\omega_{0}\right)} .
$$

The quantum dot charge susceptibility $\chi(\omega)$ can be calculated within the Keldysh formalism as

$$
\chi^{*}(\omega)=-i \int \frac{d \omega}{2 \pi} \operatorname{Tr}\left[\check{\mathcal{S}}(\omega) \check{\mathcal{G}}^{r}(\omega) \check{\Sigma}^{<}(\omega) \check{\mathcal{G}}^{a}(\omega)\right]
$$

with

$$
\check{\mathcal{S}}(\omega)=\check{\tau}\left[\check{\mathcal{G}}^{r}\left(\omega+\omega_{0}\right)+\check{\mathcal{G}}^{a}\left(\omega-\omega_{0}\right)\right] \check{\tau} .
$$

The retarded and advanced Green's functions $\check{\mathcal{G}}^{r / a}$ of the quantum dot and the lesser self-energy $\check{\Sigma}^{<}(\omega)$ are defined in Appendix B. The matrix $\check{\tau}=\operatorname{diag}(1,-1)$ describes the structure of the photon-particle coupling in the Nambu (electron-hole) space. Note that this degree of freedom is not necessary for describing the $\mathrm{N}$-dot junction, but we introduce it for later use in Sec. IV. In the present section, we disregard the $S$ reservoir and use $E_{c}=0$ so that the susceptibility $\chi(\omega)$ can be simplified as Eq. (B16) of Appendix B at zero temperature and 
○—०० - $\Delta A / A_{0}$, experiment ०—०—० $-\Delta \varphi$, experiment (rad) - $\Delta A / A_{0}$, theory - $\Delta \varphi$, theory (rad)
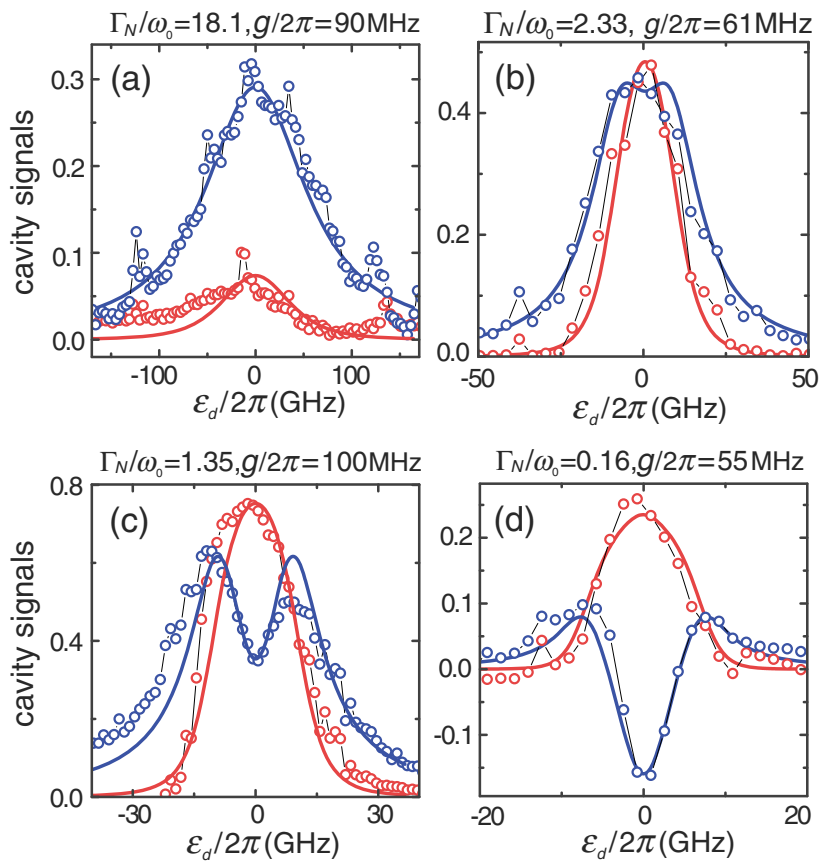

FIG. 2. Panels (a-d): Measured phase shift $\Delta \varphi$ (blue dots) and reduced amplitude shift $\Delta A / A_{0}$ (red dots) of the microwave signal transmitted by the cavity versus the energy $\varepsilon_{d}$ of the dot orbital, for $V_{b}=0$ and different dot orbitals (for clarity, we have plotted the opposite of these signals). The red and blue lines show the predictions given by Eqs. (5) and (6) for values of $\Gamma_{N}$ and $g$ given in the different panels, and $T=60 \mathrm{mK} \simeq 0.19 \omega_{0}$.

$$
\chi(\omega)=\int_{-\infty}^{+\infty} \frac{d \omega^{\prime}}{\pi \omega} \frac{\Gamma_{N}\left[f\left(\omega^{\prime}\right)-f\left(\omega^{\prime}-\omega\right)\right]}{\left(\omega^{\prime}-\varepsilon_{d}-i \frac{\Gamma_{N}}{2}\right)\left(\omega^{\prime}-\omega-\varepsilon_{d}+i \frac{\Gamma_{N}}{2}\right)},
$$

with $f(\varepsilon)=1 /\left(1+\exp \left[\varepsilon / k_{b} T\right]\right)$, for finite temperatures. Below, we interpret our data by using the exact noninteracting expression (5) of $\chi(\omega)$, which depends only on two parameters: the tunnel rate $\Gamma_{N}$ between the dot and $\mathrm{N}$, and the temperature $T$. We obtain a quantitative agreement between the measured $(\Delta \varphi, \Delta A)$ and the values calculated from the transmission ratio

$$
\left[1+\left(\Delta A / A_{0}\right)\right] e^{i \Delta \varphi}=\Lambda_{0} /\left[\Lambda_{0}-i g^{2} \chi\left(\omega_{0}\right)\right],
$$

which follows from Eq. (2) for $\omega_{\text {rf }}=\omega_{0}$ (see red and blue lines in Fig. 2). We use the same finite temperature $T=60 \mathrm{mK}$ for all the resonances. Then, for each resonance, there remains only two adjustable parameters, namely, $g$ and $\Gamma_{N}$, to simultaneously fit the $\Delta \varphi$ and $\Delta A$ curves. Remarkably, we obtain a good agreement with the data for a wide range of $\Gamma_{N} / \omega_{0}$ ratios (see Fig. 7 for supplementary resonances). The full functional form of the cavity response is accurately reproduced by our theory.
Such a modeling was not possible for previous experiments combining (real or effective) single quantum dots with microwave cavities [3,8,33].

A deeper analysis of the cavity response can be performed by studying the cavity frequency shift $\Delta \omega_{0}$ and cavity linewidth shift $\Delta \Lambda_{0}$, which can be obtained from the experimental signals $[62,63]$ as $\Delta \omega_{0}=\Lambda_{0}\left(A_{0} / A\right) \sin (\Delta \varphi)$ and $\Delta \Lambda_{0}=\Lambda_{0}\left(\left(A_{0} / A\right) \cos (\Delta \varphi)-1\right)$ and modeled theoretically from $\Delta \omega_{0}+i \Delta \Lambda_{0}=g^{2} \chi\left(\omega_{0}\right)$. To study the relation between $\Delta \Lambda_{0}$ and $\Delta \omega_{0}$, we define the ratio

$$
\theta=\frac{\pi}{2} \frac{\omega_{0}}{g^{2}} \frac{\left(\Delta \omega_{0}\right)^{2}}{\Delta \Lambda_{0}}
$$

which can be modeled theoretically as

$$
\theta=\frac{\pi}{2} \omega_{0} \frac{(\operatorname{Re}[\chi])^{2}}{\operatorname{Im}[\chi]}
$$

The dashed line in the top panel of Fig. 3 shows $\theta_{0}=$ $\theta\left(\varepsilon_{d}=0\right)$ versus $\Gamma_{N} / \omega_{0}$, calculated at $T=0$ from Eqs. (8) and (B16), for a dot level at resonance with the Fermi energy of the reservoir $\left(\varepsilon_{d}=0\right)$. Remarkably, $\theta_{0}$ shows the minimum $\theta_{0}=0$ for $\Gamma_{N} \sim 0.7 \omega_{0}$ due to the inductive to capacitive crossover of Fig. 2 . Then, in the adiabatic limit $\Gamma_{N} \gg \omega_{0}, \theta_{0}$ tends to 1 . In fact, this limit is valid for any value of $\varepsilon_{d}$, i.e.,

$$
\lim _{\Gamma_{N} / \omega_{0} \rightarrow+\infty} \theta\left(\varepsilon_{d}\right)=1
$$

The solid gray line in Fig. 3, top panel, shows $\theta_{0}$ for the temperature $T=60 \mathrm{mK}$, calculated from Eqs. (5) and (8). It illustrates that finite temperatures quantitatively affect the behavior of the system for low values of $\Gamma_{N}$, but Eq. (9) remains valid as long as $\Gamma_{N} \gg k_{B} T$. A straightforward question is whether the nontrivial behavior of Eq. (9) can be observed with our experiment. This equation has two important implications. First, it predicts that the $\Delta \Lambda_{0}$ and $\left(\Delta \omega_{0}\right)^{2}$ curves versus $\varepsilon_{d}$ (or, equivalently, versus the dot gate voltage $V_{g}$ ) should be proportional in the open contact limit. Second, it gives the exact value of the proportionality constant between $\Delta \Lambda_{0}$ and $\left(\Delta \omega_{0}\right)^{2}$. The latter cannot be accessed in our experiment. Indeed, we cannot calibrate the absolute value of $\theta$ because we do not have an independent experimental determination of the parameter $g$. Instead, we determine $g$ and thus $\theta$ from a fitting procedure that relies on the assumptions of our theory. Nevertheless, we can experimentally test the scaling between $\Delta \Lambda_{0}$ and $\left(\Delta \omega_{0}\right)^{2}$, as discussed below.

To illustrate the large variety of regimes achieved with our experiment, we show with crosses the fitted values of $\theta_{0}$, calculated from Eqs. (5) and (8), for the fitting parameter $\Gamma_{N}$ of the different resonances in Figs. 2 and 7 and $T=60 \mathrm{mK}$. In principle, the scaling between 

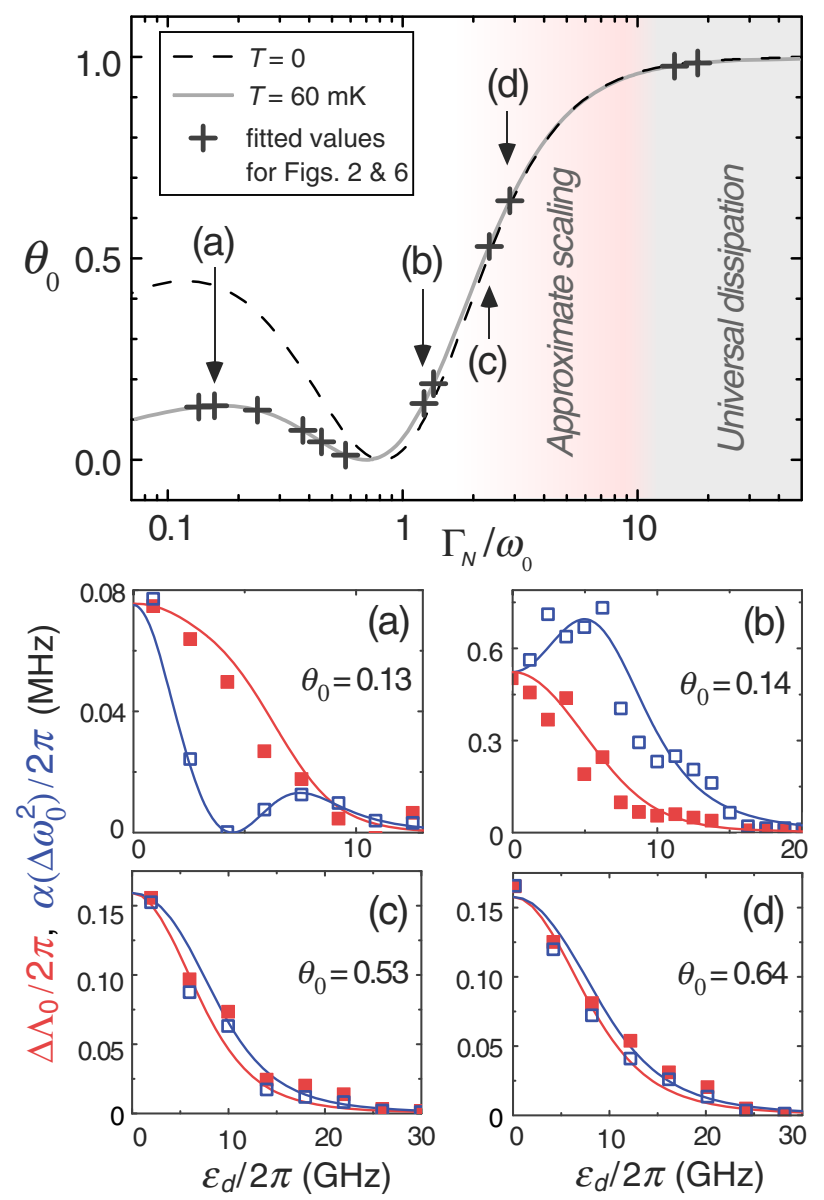

FIG. 3. Top panel: Ratio $\theta_{0}=\theta\left(\varepsilon_{d}=0\right)$ versus the tunnel rate $\Gamma_{N}$, calculated from Eqs. (5), (8), and (B16) for $T=0$ (black dashed line) and $T=60 \mathrm{mK}$ (gray solid line). The crosses correspond to fitted values of $\theta_{0}$, calculated from Eqs. (5) and (8) for the different resonances in Figs. 2 and 7. Bottom panels: Comparison between the experimental $\Delta \Lambda_{0}$ and $\left(\Delta \omega_{0}\right)^{2}$, using the scaling factor $\alpha=\pi \omega_{0} / 2 \theta_{0} g^{2}$, with $\theta_{0}$ indicated with arrows in the top panel. We use $\Gamma_{N} / \omega_{0}=0.16,1.23,2.33$, and 2.86 from left to right and top to bottom panels. We also show, as blue and red solid lines, the calculated $\Delta \Lambda_{0}$ and $\left(\Delta \omega_{0}\right)^{2}$.

$\Delta \Lambda_{0}$ and $\left(\Delta \omega_{0}\right)^{2}$ should be closely satisfied in the gray area where $\theta_{0} \simeq 1$. However, for the two resonances we have found in this area $\left(\Gamma_{N}=18.1 \omega_{0}\right.$ and $\left.\Gamma_{N}=14.3 \omega_{0}\right)$, we cannot reliably determine $\Delta \Lambda_{0}$ from $\Delta A$ and $\Delta \varphi$ because $\Delta A$ is small and thus affected too much by background variations. This difficulty arises because in the adiabatic limit, the dot charge is in phase with the dot gate excitation, i.e., $\chi\left(\omega_{0} \ll \Gamma_{N}\right)=\hbar \partial\langle n\rangle / \partial \varepsilon_{d} \in \mathbb{R}$, with $\langle n\rangle$ the static charge occupation of the dot. This is why, for $\Gamma_{N} \gg \omega_{0}$, we find that $\Delta \Lambda_{0}=g^{2} \operatorname{Im}\left[\chi\left(\omega_{0}\right)\right] \sim$ $8 g^{2}\left(\omega_{0} / \Gamma_{N}\right)^{2} / \pi \omega_{0}$ vanishes like $\left(\omega_{0} / \Gamma_{N}\right)^{2}$. From Ref. [63], $\Delta A$ is itself small in this case. Nevertheless, we can interpret the raw cavity signals $\Delta \varphi$ and $\Delta A$ with the same theory as our other data, which shows that they are consistent with the universality of charge relaxation [see
Figs. 2(a) and 7]. To resolve the scaling behavior of the cavity response, we now consider the resonances at $\Gamma_{N}=$ $2.40 \omega_{0}$ and $\Gamma_{N}=2.86 \omega_{0}$. These points belong to the pink area $2 \leq \Gamma_{N} \lesssim 10$ of Fig. 3, where, from our theory, the scaling behavior should still hold approximately, although $\theta_{0}<1$. As visible in Figs. 3(c) and 3(d), we indeed find that the proportionality between the experimental $\Delta \Lambda_{0}$ and $\left(\Delta \omega_{0}\right)^{2}$ is satisfied to a good accuracy, with a scaling factor $\alpha=\pi \omega_{0} / 2 \theta_{0} g^{2}$. Small discrepancies between $\Delta \Lambda_{0}$ and $\alpha\left(\Delta \omega_{0}\right)^{2}$ are visible in the theoretical curves (see red and blue lines) but not resolvable experimentally. Such a scaling behavior is observed here for the first time. Finally, we can observe how the scaling behavior breaks down for smaller tunnel rates. When $\Gamma_{N}$ decreases, the $\left(\Delta \omega_{0}\right)^{2}$ peak versus $\varepsilon_{d}$ first becomes wider than the $\Delta \Lambda_{0}$ peak (not shown) before becoming strongly nonmonotonic [see Figs. 3(a) and 3(b)].

The remarkable scaling between $\Delta \Lambda_{0}$ and $\left(\Delta \omega_{0}\right)^{2}$ is directly related to the universality of the ac resistance of a quantum dot circuit, which was predicted by M. Büttiker et al. two decades ago $[37,38]$ and recently revisited as a Korringa Shiba relation $[43,45,64]$. More precisely, for a noninteracting $\mathrm{N}$-dot junction $\left(E_{c}=0\right)$ excited at a frequency $\omega_{\mathrm{rf}}$ such that $\Gamma_{N} \gg \omega_{\mathrm{rf}}, k_{B} T$, the ac resistance is set by $\theta\left(\varepsilon_{d}\right)$, i.e., $R_{\mathrm{ac}}=h / 4 e^{2} \theta\left(\varepsilon_{d}\right)$, which gives $R_{\mathrm{ac}}=h / 4 e^{2}$ for our spin-degenerate case, for any gate voltage. This effect can be understood as a quantum charge relaxation effect, which involves the internal coherent dynamics of the quantum dot. So far, the universality of the quantum charge relaxation has been observed only with a strongly spinpolarized GaAs two-dimensional electron gas device [39]. Here, we present the second example of system, i.e., a spindegenerate carbon nanotube device, whose behavior is consistent with this phenomenon. Indeed, the scaling behavior between $\Delta \Lambda_{0}$ and $\left(\Delta \omega_{0}\right)^{2}$ reveals the independence of $R_{\mathrm{ac}}$ from the dot gate voltage $V_{g}$ (or, equivalently, from the dot orbital energy $\varepsilon_{d}$ ), a property that could not be probed in Ref. [39] and that is already valid for intermediate tunnel rates.

The fact that we model the charge susceptibility of the quantum dot circuit with a noninteracting model in spite of Coulomb blockade is nontrivial. This approach is useful to understand our data because we are in a deep Coulomb blockade regime $\left(\Gamma_{N} \ll E_{c}\right)$, where correlation effects induced by interactions (e.g., the Kondo effect) are weak. In this limit, one can expect $G$ and $\chi$ to show variations similar to those of the noninteracting case, with only quantitative modifications. In particular, a reduction of the amplitude of the signals is expected because of the reduction of the dot occupation by Coulomb blockade [65]. Our results suggest that in our regime of parameters, interactions simply lead to a renormalization of our fitting parameters. In principle, it is possible to generalize our model to the interacting case to quantitatively study the effects of a finite $E_{c}[65,66]$. This is beyond the scope of the 
present article. Our observation of the scaling between $\Delta \Lambda_{0}$ and $\left(\Delta \omega_{0}\right)^{2}$ is independent of any theoretical assumption on the dot interaction regime since the calculation of these quantities from the raw data only relies on Eq. (2). The fact that we observe the scaling behavior between $\Delta \Lambda_{0}$ and $\left(\Delta \omega_{0}\right)^{2}$ in spite of a strong Coulomb blockade is remarkable. This is in agreement with recent theory works that suggest that the universality of the charge relaxation resistance $R_{\mathrm{ac}}$ persists in the spin-degenerate interacting case $[45,46]$.

\section{NEGATIVE PHOTON DAMPING BY A N-DOT-S BIJUNCTION}

A common belief is that a fermionic reservoir should necessarily damp cavity photons since it calls for irreversible processes. Is it possible to go against this natural trend? To answer this question, we consider the finite bias voltage regime $V_{b} \neq 0$, where our device implements a $\mathrm{N}$-dot-S bijunction. This can be confirmed from the bijunction conductance versus $V_{b}$ and the dot gate voltage $V_{g}$ [Fig. 4(a)]. Like in Fig. 1(d), we observe two Coulomb triangles that do not close on the $V_{b}=0$ line but at $e V_{b} \sim \pm \Delta$, and that are shifted along the $V_{g}$ axis. These features are typical of a N-dot-S structure and are due to the gap and BCS peaks in the density of states of the $\mathrm{S}$ contact [67-69]. The conductance resonances corresponding to an alignment between the dot level and the BCS peaks display negative differential resistance areas [68] [see red areas in Fig. 4(a)]. This can be understood easily in the limit $\Gamma_{S} \ll k_{B} T$, where, from a Fermi's golden rule argument, the conductance is proportional to the derivative of the BCS peak [70]. It is also interesting to notice that the conductance above the gap has a small amplitude $|G|<0.12 \times e^{2} / h$, which suggests a strong asymmetry between the tunnel rates $\Gamma_{N}$ and $\Gamma_{S}$ to the $N$ and $S$ contacts. A theoretical modeling of the conductance with Eq. (B17) of Appendix B confirms that for the dot level considered in this section, one has $\Gamma_{S} \ll \Gamma_{N}<k_{B} T$ [see Fig. 4(b)].

We have measured the cavity signals simultaneously with $G$ [Figs. 4(c) and 4(e)]. In agreement with Sec. III, $\Delta \varphi$ and $\Delta A$ reveal the resonance between the dot level and the Fermi energy of the $\mathrm{N}$ contact even inside the gap area $\left(e\left|V_{b}\right|<\Delta\right)$, in contrast to what happens for $G$. Sign changes in $\Delta \varphi$ similar to those of Fig. 2(d) indicate that we are in a regime with $\Gamma_{S}, \Gamma_{N} \ll \omega_{0}$. The microwave amplitude $A$ shows a more surprising behavior. Indeed, the resonances of the dot level with $\mathrm{S}$ and $\mathrm{N}$ do not affect the $A$ signal in a similar way. For $e\left|V_{b}\right|>\Delta$, the resonances with the $\mathrm{S}$ contact are closely followed by an area with $\Delta A>0$, which indicates a counterintuitive negative photon damping (or photon emission) caused by a fermionic reservoir [see dark blue areas in Fig. 4(e)]. So far, with quantum dot circuits coupled to cavities, photon emission has been obtained only due to tunneling between two discrete dot levels [13-16].
To model the cavity response, we again use Eqs. (3) and (6), with expressions of $\check{\mathcal{G}}^{r / a}(\omega)$ and $\check{\Sigma}^{<}(\omega)$ that take into account the finite $\Gamma_{S}$ [see Eqs. (B9)-(B15) of Appendix B]. We can quantitatively reproduce the three signals $\Delta \varphi, \Delta A$, and $G$ versus $V_{b}$ and $V_{g}$ with a consistent set of parameters [see Figs. 4(b), 4(d), and 4(f)]. The good agreement between the data and theory is also visible in Fig. 8 of Appendix A for constant values of $V_{b}$. In particular, our theory reproduces well the positive $\Delta A$
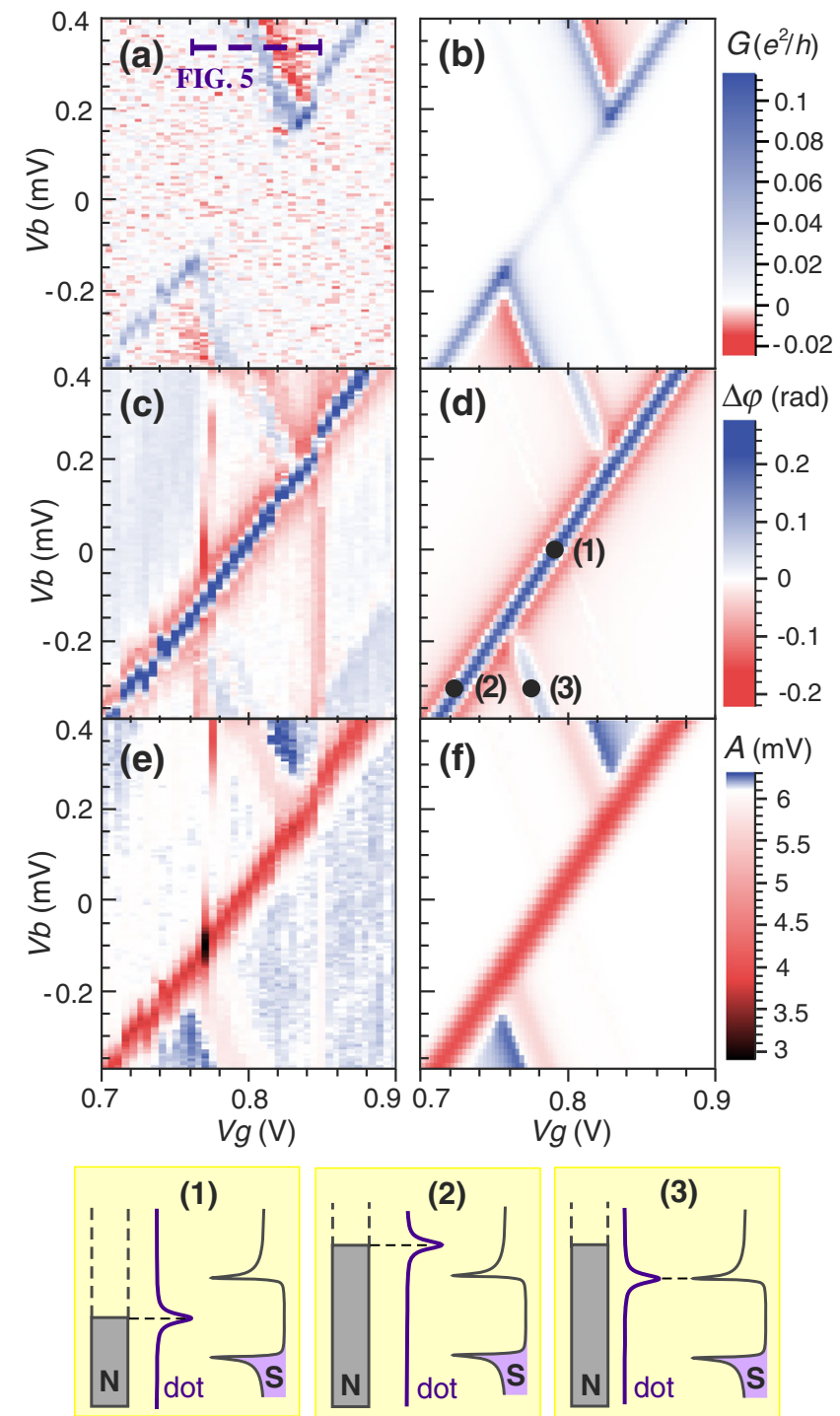

FIG. 4. Panels (a), (c), and (e): Measured linear conductance $G$, phase shift $\Delta \varphi$, and total amplitude $A$ of the transmitted microwave signal versus the dot gate voltage $V_{g}$ and the bias voltage $V_{b}$. Panels (b), (d), and (d): Predictions from Eqs. (3), (6), and (B17), for $\Gamma_{N} / 2 \pi=0.6 \mathrm{GHz}, \quad \Gamma_{S} / 2 \pi=65 \mathrm{MHz}, \quad \Gamma_{n} / 2 \pi=$ $8 \mathrm{GHz}, g / 2 \pi=99 \mathrm{MHz}, \Delta=0.17 \mathrm{meV}, T=90 \mathrm{mK}, \omega_{0} / 2 \pi=$ $6.65 \mathrm{GHz}, A_{0}=6.1 \mathrm{mV}$, and $\Lambda_{0} / 2 \pi=0.259 \mathrm{MHz}$. The white color corresponds to $A=A_{0}$ in panels (e) and (f). Panels (1), (2), and (3): Electric potential configuration corresponding to the black points in panel (d). 
areas. We take into account the lever arms determining the shift of the dot and reservoir energy levels with $V_{b}$ and $V_{g}$. We also use the gap value $\Delta=0.17 \mathrm{meV}$ given straightforwardly by the $G\left(V_{b}, V_{g}\right)$ map [69]. Then, only five adjustable parameters remain: $\Gamma_{N}, \Gamma_{S}, g, T$, and the broadening parameter $\Gamma_{n}$ for the BCS peaks. Simultaneously fitting three two-dimensional plots in these conditions is nontrivial and possible only because of the adequacy of our model. The agreement with the data is optimal for $\quad \Gamma_{N} / 2 \pi=0.6 \mathrm{GHz}, \quad \Gamma_{S} / 2 \pi=65 \mathrm{MHz}$, $\Gamma_{n} / 2 \pi=8 \mathrm{GHz}, \quad g / 2 \pi=99 \mathrm{MHz}, \quad$ and $T=90 \mathrm{mK}$. Equation (B17), used to model $G$, has been obtained in the absence of the cavity $(g=0)$. This approximation is relevant because the cavity brings only small corrections to this expression, not resolvable in our experiment. In contrast, $\Delta \varphi$ and $A$ are calculated to second order in $g$. We have again used a noninteracting approach to model the dot behavior. This approximation is relevant to understand our data because we are in the deep Coulomb blockade regime and because Andreev reflections (which are very sensitive to interactions) are negligible in the small $\Gamma_{S}$ limit. Therefore, interactions should only induce quantitative modifications of the dot-lead resonances. Note that a temperature $T=60 \mathrm{mK}$ is optimal to interpret the low bias voltage data of Sec. III, but we need a higher temperature $T=90 \mathrm{mK}$ to interpret the finite bias voltage data of Sec. IV. This may be due to heating effects caused by $V_{b} \neq 0$ or to interactions that can modify the dot occupation and thus the amplitude of dot-lead resonances in the out-of-equilibrium regime.

Are the $G<0$ and $\Delta A>0$ effects related? In order to answer this question, Fig. 5 shows the measured $A$ and $G$ versus $V_{g}$ (red dots) together with the theory of Fig. 4 (red lines), for a constant bias voltage $V_{b}=0.336 \mathrm{mV}$, along the dashed line in Fig. 4(a). These signals vary smoothly due to the large value of $\Gamma_{n}$. It is very instructive to use a smaller BCS peak broadening parameter $\Gamma_{n} / 2 \pi=1 \mathrm{GHz}$ for the theory (blue lines). The $A$ signal then shows a cusp when the dot level is at resonance with a BCS peak [gray dashed line (2)] or shifted by $\pm \hbar \omega_{0} / \alpha$ [gray dashed lines (1) and (3)], with $\alpha$ the lever arm associated to $V_{g}$. This indicates inelastic tunneling accompanied by photon absorption or emission along lines (1) and (3). More precisely, in the configuration corresponding to panel (1) or (3) of Fig. 5, the BCS peaks of the S contact reinforce the probability of photon absorption or emission, leading to a pronounced negative or positive $\Delta A$ peak. In contrast, one keeps $\Delta A<0$ near the $\mathrm{N}$-dot resonance because the density of states of the $N$ contact can be considered as constant. As expected, the theoretical $G$ for $\Gamma_{n} / 2 \pi=1 \mathrm{GHz}$ and $g=0$ does not show cusps along lines (1) and (3) since this quantity does not take into account photon emission and absorption (see blue line in top panel of Fig. 5). Since the experimental $G$ is dominated by the zeroth-order contribution in $g$, it implies that the
$G<0$ and $\Delta A>0$ effects in our data are not directly related. It is more correct to state that these two effects have a common origin. More precisely, $G<0$ is due to the fact that the DOS of $S$ decreases with energy in certain areas, which leads to a reduction of the dot current, whereas $\Delta A>0$ is due to the strong DOS peaks that reinforce photon emission. Note that Fig. 1 shows extra resonant lines parallel to the Coulomb diamond borders, which can be attributed to excited states of the quantum dot. The excitation energy $E_{\text {ex }}$ of these levels is such that $E_{\mathrm{ex}} \gtrsim 0.30 \mathrm{meV} \gg \hbar \omega 0 \simeq 0.027 \mathrm{meV}$. Furthermore, the measurements of Sec. IV have been realized in another gate voltage range where such excited states are not visible. Hence, the dot excited states can be disregarded to discuss photon-assisted tunneling.

It is important to replace the above results in a wider context. Photon-assisted tunneling has been observed for 40 years in SIS junctions [71] and for 25 years in quantum dots with $\mathrm{N}$ contacts [72-74]. In these seminal experiments, a broadband coupling scheme was used instead of a cavity, and the photo-induced current was directly measured. In
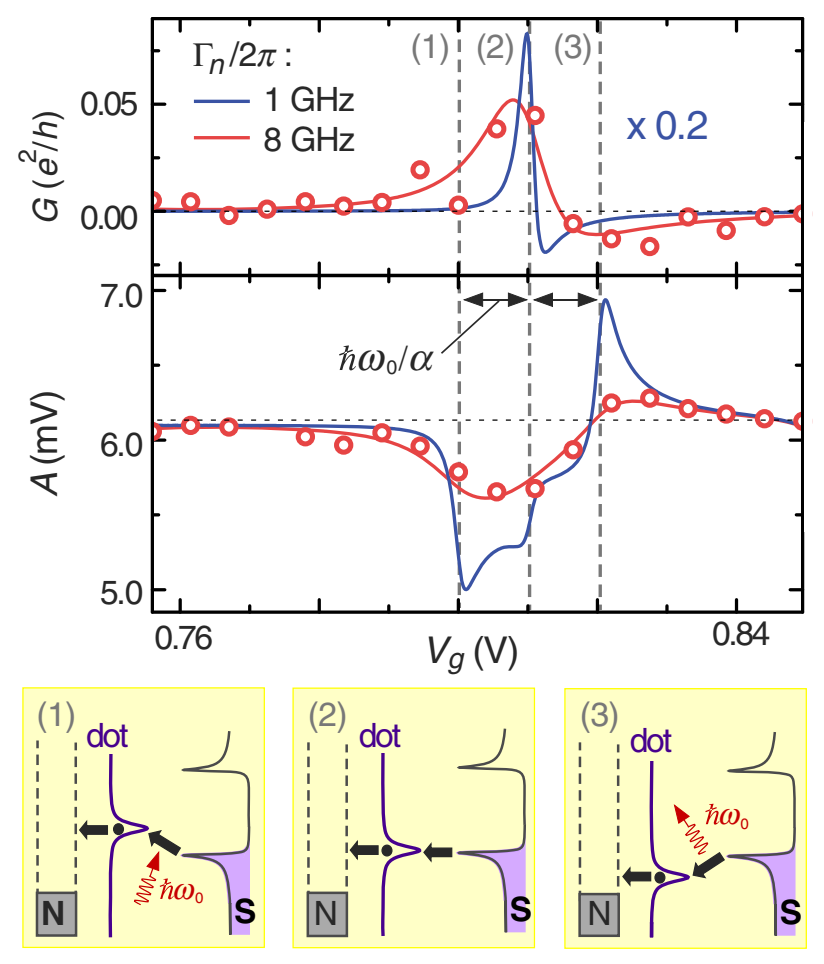

FIG. 5. Conductance $G$ and microwave amplitude $A$ versus $V_{g}$ (red dots), measured along the dashed line in Fig. 4(a) for $V_{b}=0.336 \mathrm{mV}$, and theory using the same parameters as in Fig. 4 and $\Gamma_{n} / 2 \pi=8 \mathrm{GHz}$ (red lines) or $\Gamma_{n} / 2 \pi=1 \mathrm{GHz}$ (blue lines). The theoretical $G$ for $\Gamma_{n} / 2 \pi=1 \mathrm{GHz}$ has been multiplied by 0.2 . Panels (1), (2), and (3) illustrate the transport regimes corresponding to the gray dashed lines. In panel (2), the dot orbital is resonant with a BCS peak in the DOS of the $\mathrm{S}$ reservoir. In panels (1) and (3), an electron can pass from the dot orbital to a BCS peak by absorbing or emitting a cavity photon. 
this context, one novelty of our work is that we use a highly resonant microwave technique to inject and probe photons. We send a limited photonic power on the quantum dot circuit, so the photo-induced current is not resolvable. More precisely, along lines (1) and (3), the rate of photon absorption or emission $\left(\Gamma_{e / a}\right)$ by the quantum dot circuit is $\Gamma_{e / a} \simeq 2 n_{p h} \Lambda_{0} \Delta A / A_{0}$, with an average photon number $n_{p h} \sim 120$ in the cavity [75]. This gives $\Gamma_{e / a} \sim 2 \mathrm{MHz}$, which corresponds to a photon-assisted current of the order of $0.3 \mathrm{pA}$. For comparison, in Ref. [72], the photon-assisted current between a dot and a $\mathrm{N}$ contact reaches $30 \mathrm{pA}$. In spite of this, we can directly detect photon emission and absorption thanks to the cavity. This demonstrates that circuit QED techniques provide accurate tools to revisit the physics of photon-assisted tunneling. Note that despite longstanding theoretical interest [50-59], our work represents the first experimental study of photon-assisted tunneling between a quantum dot and a superconductor.

\section{SUMMARY, EXTENSION OF OUR THEORY, AND PERSPECTIVES}

We have experimentally studied the behavior of a spindegenerate N-dot-S hybrid structure based on a carbon nanotube, coupled to a microwave cavity with frequency $\omega_{0}$. We have observed a large variety of effects depending on the values of the tunnel rates and on the bias voltage applied to the device. For intermediate $\mathrm{N}$-dot tunnel rates $\Gamma_{N} \sim 2.5 \omega_{0}$ and equilibrium conditions, the cavity frequency and linewidth shifts follow a scaling relation that is independent of the quantum dot gate voltage. This behavior is related to the universality of the quantum charge relaxation resistance $R_{\mathrm{ac}}$ predicted by Büttiker and coworkers [37,38] in the adiabatic limit $\left(\omega_{0} \ll \Gamma_{N}\right)$. More precisely, it reveals the independence of $R_{\text {ac }}$ from the dot gate voltage, which is already approximately valid for intermediate tunnel rates $\Gamma_{N} \sim 2.5 \omega_{0}$. Remarkably, we obtain this behavior in spite of the presence of Coulomb blockade in the dot, which was not taken into account in the original model by Büttiker et al. This observation is consistent with recent theory works that predict that the universality of charge relaxation should persist in the spindegenerate interacting case $[45,46]$. Our measurements are doubly complementary to those of Gabelli et al., who have observed the universal charge relaxation in the (spinpolarized) noninteracting case and who could not probe the gate dependence of $R_{\text {ac }}$ [39]. We have also observed, in a controlled way, the departure from the scaling regime, when the dot behavior changes from capacitive to inductive. In the finite bias voltage regime, we have observed negative photon damping by the quantum dot circuit. This reveals photon emission caused by inelastic quasiparticle tunneling between the dot and the BCS peaks of the S reservoir. The cavity signals are able to reveal this process, although it is not resolvable in the dot dc current. Strikingly, all the effects depicted above can be modeled quantitatively with a single noninteracting description. Hence, in our regime of parameters, strong Coulomb blockade, which we have disregarded, does not seem to modify the main physical behavior of our system. The agreement between our data and theory suggests that interactions simply lead to a renormalization of our fitting parameters. Nevertheless, a comparison between our data and a fully interacting theory would be interesting. So far, theory works have mainly focused on the value of $R_{\mathrm{ac}}$ for $V_{b}=0$, but our work shows that the cavity frequency shift and linewidth shift deserve to be studied independently in the full $V_{g}$ and $V_{b}$ ranges. More generally, our work validates a description of mesoscopic QED experiments in terms of an electronic charge susceptibility.

Considering the agreement of our theory with experimental data, it is interesting to generalize it to more complex hybrid structures. The versatility of nanofabrication techniques allows us to envision a large variety of experiments combining quantum dot circuits and cavities. In practice, nanoconductors can be tunnel-coupled to various types of fermionic reservoirs such as normal metals and superconductors [76], but also ferromagnets with collinear [18,77] or noncollinear magnetizations [17,78]. These different elements can be combined in a large variety of geometries, involving, for instance, interdot hopping [6-17] and multiterminal contacting [77,79]. In this context, we generalize our approach to geometries with several quantum dots or sites or several orbitals. In the case where each discrete level $i \in[1, N]$ of the nanocircuit is shifted by the cavity field $\hat{a}+\hat{a}^{\dagger}$ with a constant $g_{i}$, we obtain

$$
\frac{b_{t}}{b_{i n}}=\frac{t_{0}}{\omega_{\mathrm{rf}}-\omega_{0}-i \Lambda_{0}-\Sigma_{i, j} g_{i} g_{j} \chi_{i j}\left(\omega_{0}\right)} .
$$

In the linear response limit, the susceptibility $\chi_{i j}(\omega)$ for orbital indices $i, j$ can be calculated within the Keldysh formalism as [80]

$$
\chi_{i, j}^{*}\left(\omega_{0}\right)=-i \int \frac{d \omega}{2 \pi} \operatorname{Tr}\left[\check{\mathcal{S}}_{i j}(\omega) \check{\mathcal{G}}^{r}(\omega) \check{\Sigma}^{<}(\omega) \check{\mathcal{G}}^{a}(\omega)\right],
$$

with

$$
\check{\mathcal{S}}_{i j}(\omega)=\check{\tau}_{i} \check{\mathcal{G}}^{r}\left(\omega+\omega_{0}\right) \check{\tau}_{j}+\check{\tau}_{j} \check{\mathcal{G}}^{a}\left(\omega-\omega_{0}\right) \check{\tau}_{i}
$$

These expressions involve multisite Keldysh Green's functions $\check{\mathcal{G}}^{r(a)}$, a lesser self-energy $\check{\Sigma}^{<}$, and the electron-photon coupling element $\check{\tau}_{i}$ at site $i$, which are defined in Appendix B 4. In principle, the susceptibility $\chi_{i j}(\omega)$ can be calculated with techniques other than the Keldysh formalism [81,82]. However, one interesting feature of this approach is that it is particularly convenient for describing nonequilibrium configurations with multiple 
quantum dots and multiple reservoirs (normal metals, ferromagnets, and superconductors), as illustrated, for instance, by Ref. [83]. It goes beyond the sequential tunneling picture used so far to interpret most mesoscopic QED experiments. In principle, it also enables the description of Coulomb interactions $[65,66]$.

The above formalism could be instrumental for understanding the behavior of complex cavity-nanocircuit hybrid structures. In particular, it is suitable for understanding the interaction between cavity photons and Cooper pair splitters, or topological hybrid nanocircuits. Nonlocal entanglement and self-adjoint Majorana bound states are intensively sought after in these devices, and new investigation tools such as cavity photons could be instrumental in this quest. More generally, our results show that mesoscopic QED represents a powerful toolbox to investigate quantum charge relaxation, photon-assisted tunneling, and all other effects involving tunneling between a discrete level and fermionic reservoirs. This opens many possibilities. For instance, the dynamics of the many-body Kondo effect could be explored thanks to circuit QED techniques. Quantum dot circuits could also open new possibilities for the so-called "quantum reservoir engineering" [84,85], which would exploit fermionic reservoirs in nonequilibrium configurations to prepare nontrivial photonic and electronic states. Finally, there is a direct analogy between our setup and a quantum dot circuit coupled to the vibrational modes of a nano-object [86]. Hence, our findings could be transposed to understand the dissipation of nano-electromechanical systems.

\section{ACKNOWLEDGMENTS}

We acknowledge useful discussions with M. Büttiker, T. Cubaynes, R. Deblock, G. Fève, and F. Mallet. This work was financed by the ERC Starting grant CirQys, the EU FP7 Project No. SE2ND[271554], and the ANRNanoQuartet [ANR12BS1000701] (France).

\section{APPENDIX A: EXPERIMENTAL DETAILS}

\section{Sample fabrication and control}

The cavity is a half-wavelength superconducting $\mathrm{Nb}$ transmission line resonator, with a bare frequency $\omega_{0} / 2 \pi \sim$ $6.65 \mathrm{GHz}$ and a quality factor $Q \sim 12800$. We measure the cavity transmission $b_{t} / b_{i n}$ with a homodynelike detection scheme. To form the quantum dot circuit, we use a carbon nanotube grown by chemical vapor deposition, which is stamped into the cavity to preserve $Q$ [87]. On the nanotube, we evaporate two $\mathrm{N}$ contacts formed by $70 \mathrm{~nm}$ of $\mathrm{Pd}$, and a $\mathrm{S}$ contact formed by $4 \mathrm{~nm}$ of Pd proximized with $100 \mathrm{~nm}$ of Al. The nanotube sections on the left and right of the $\mathrm{S}$ contact are coupled to remote dc gates with voltages $V_{g}^{L}$ and $V_{g}^{R}$ [see Fig. 1(a)]. The ac top gate (TG) consists of a trilayer $\mathrm{Al}_{2} \mathrm{O}_{3}(6 \mathrm{~nm}) / \mathrm{Al}(50 \mathrm{~nm}) / \mathrm{Pd}(20 \mathrm{~nm})$. The double dot design of our sample was initially developed for a
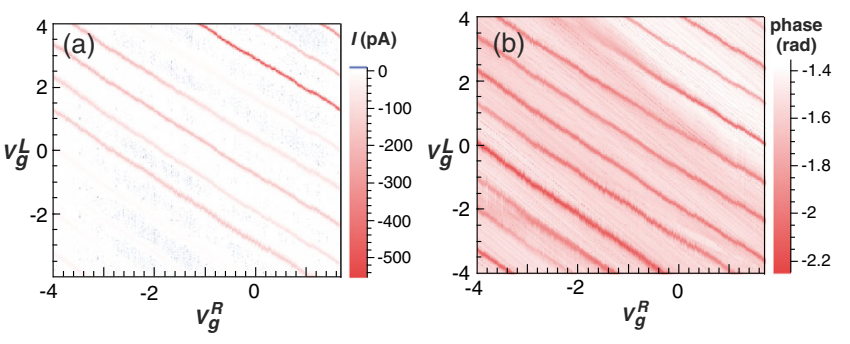

FIG. 6. Current $I$ through the quantum dot (left panel) and cavity signal $\Delta \varphi$ (right panel) versus $V_{g}^{L}$ and $V_{g}^{R}$, in the area corresponding to the data of Figs. 3(c) and 3(d).

Cooper pair splitting experiment, which will be reported elsewhere [88].

The sample is placed in a dilution refrigerator with a base temperature of $16 \mathrm{mK}$. We apply the same bias voltage $V_{b}$ to the two $\mathrm{N}$ contacts. We measure the current $I$ in the $\mathrm{S}$ contact with a dc measurement, and we use a lock-in detection to determine the corresponding differential conductance $G$. For $e\left|V_{b}\right|>\Delta$, the current $I$ versus $V_{g}^{L}$ and $V_{g}^{R}$ and the cavity signals correspond to a pattern of parallel lines (see Fig. 6). This indicates that the whole nanotube section between the two $\mathrm{N}$ contacts behaves as a single quantum dot. Therefore, in Sec. IV, we use an effective gate voltage parameter $V_{g}=a_{L} V_{g}^{L}+a_{R} V_{g}^{R}$ to represent the data. In Sec. III, we use level-dependent lever arms $\alpha$ and gate voltage offsets $V_{g}^{0}$ to express the gate voltage axis in terms of the energy $\varepsilon_{d}=\alpha\left(V_{g}-V_{g}^{0}\right)$ of the considered dot level with respect to the Fermi energy of the $\mathrm{N}$ reservoir (see Table I for the values of the parameters $\alpha$ and $V_{g}^{0}$ ). This is more convenient to compare the energy width of the different resonances.

\section{Supplementary data and system parameters}

In order to further demonstrate the quantitative agreement between our theoretical approach and the data, we present supplementary data together with their theoretical modeling. Figure 7 shows the cavity signals $\Delta \varphi$ and $\Delta A$ at $V_{b}=0$ for 12 different quantum dot-reservoir resonances, including those of Figs. 2 and 3 for completeness. Figure 8 shows the dot conductance and cavity signals, for different values of $V_{b}$, on a wider $V_{g}$ scale than in Fig. 5.

Near each dot-reservoir resonance, we calibrate the bare cavity linewidth $\Lambda_{0} \sim 2 \pi \times 0.26 \mathrm{MHz}$ and the bare cavity transmission amplitude $A_{0} \sim 6.1 \mathrm{mV}$. The average photon number $n_{p h}$ in our measurements is estimated from setup transmission calibration. Assuming a $6 \mathrm{~dB}$ uncertainty, we obtain a lower bound $n_{p h}>20$, which ensures the validity of the semiclassical approximation used in our theory (see Appendix B). The agreement between our theory and data also confirms that we remain in the linear response regime invoked in Appendix B. Otherwise, the width of the 
TABLE I. Parameters corresponding to the different N-dot resonances shown in Fig. 7. We first show the effective gate voltage $V_{g}^{0}$ and the lever arm $\alpha$ extracted from the experimental data, and the fitting parameters $\Gamma_{N}$ and $g$ used to model the cavity signals $\Delta \varphi$ and $\Delta A$. We also show, when possible, the value of the $\mathrm{N}$-dot tunnel rate $\widetilde{\Gamma_{N}}$ estimated from the conductance data through the $\mathrm{S}$-dot-N structure for a voltage $\tilde{V}_{b}>\Delta / e$. Each block in the table corresponds to the one shot measurement of a given gate voltage range. The signals $\Delta \varphi$, $\Delta A$, and $G$ were measured simultaneously in each measurement shot.

\begin{tabular}{lllllllllllll}
\hline \hline Measurement shot & \multicolumn{1}{c}{1} & \multicolumn{1}{c}{1} & \multicolumn{1}{c}{2} & \multicolumn{1}{c}{3} & \multicolumn{1}{c}{3} & \multicolumn{1}{c}{3} & \multicolumn{1}{c}{4} & 5 & 6 & 6 & 6 \\
\hline$V_{g}^{0}(\mathrm{~V})$ & -0.88 & 0.09 & 0.67 & -1.30 & -0.65 & 0.47 & 0.75 & 1.16 & -2.03 & -1.41 & -0.72 & -0.16 \\
$\alpha / 2 \pi\left(\mathrm{GHz} . \mathrm{V}^{-1}\right)$ & 200 & 200 & 667 & 629 & 629 & 629 & 657 & 765 & 704 & 704 & 704 & 704 \\
$g / 2 \pi(\mathrm{MHz})$ & 69 & 61 & 55 & 100 & 83 & 60 & 66 & 70 & 68 & 120 & 85 & 90 \\
$\Gamma_{N} / \omega_{0}$ & 2.86 & 2.33 & 0.16 & 1.35 & 1.23 & 0.14 & 0.57 & 0.38 & 0.45 & 0.24 & 14.3 & 18.1 \\
$\Gamma_{N} / 2 \pi(\mathrm{GHz})$ & 19 & 15.5 & 1.05 & 9.00 & 8.20 & 0.9 & 3.8 & 2.5 & 3 & 1.6 & 95 \\
$\widetilde{\Gamma_{N} / 2 \pi(\mathrm{GHz})}$ & 21 & 16 & $\times$ & 12.5 & 7.5 & $\times$ & $\times$ & $\times$ & $\times$ & $\times$ & 64 & 120 \\
$\tilde{V}_{b}(\mathrm{mV})$ & -0.18 & -0.37 & $\times$ & 0.42 & 0.32 & $\times$ & $\times$ & $\times$ & $\times$ & $\times$ & 0.24 & 0.24 \\
\hline \hline
\end{tabular}

resonances in the cavity response would not match with the theory [89].

The parameters $V_{g}^{0}, \alpha, \Gamma_{N}$, and $g$ for the 12 resonances presented in Figs. 2 and 7 are given in the bottom table of Fig. 7. The dot-photon coupling $g$ varies from $2 \pi \times 55 \mathrm{MHz}$ to $2 \pi \times 120 \mathrm{MHz}$ and the tunnel rate $\Gamma_{N}$ from $2 \pi \times 0.9 \mathrm{GHz}$ to $2 \pi \times 120 \mathrm{GHz}$. The circuit parameters may take different values for different measurement shots, probably due to charge reorganizations in the sample, which change the offset $V_{g}^{0}$ or the potential landscape of the quantum dot. Therefore, we have separated Table I into different blocks that correspond to single-shot measurements of a given gate voltage range. The tunnel rate $\Gamma_{N}$ does not show a monotonic dependence with $V_{g}$ on a large scale. Sometimes, we find a locally monotonic dependence, on a scale of about 3 consecutive resonances, as illustrated by the blocks corresponding to measurements 3 and 6 in the Table I. The nonmonotonic behavior of $\Gamma_{N}$ with $V_{g}$ is very common in carbon nanotubes and may be attributed to weak disorder. Moreover, the variations of $\Gamma_{N}$ and $g$ do not seem correlated, probably because $\Gamma_{N}$ depends on the properties of the dot interfaces whereas $g$ depends on the overlap of the whole dot orbital with the cavity photonic pseudopotential [5]. Finally, the value of $\alpha$ for the measurement 1 differs significantly from the values used in the other measurements because $a_{L}=1$ and $a_{L}=0$ were used for measurement 1 whereas $a_{L}=0.75$ and $a_{R}=0.66$ were used for the other measurements. In Sec. IV, we have used, in the theory, $\varepsilon_{d}=\alpha\left(V_{g}-V_{g}^{0}\right)+\gamma V_{b}$ with $V_{g}^{0}=0.79 \mathrm{~V}$, $\alpha=2 \pi \times 723 \mathrm{GHz} . \mathrm{V}^{-1}$ and $\gamma=2 \pi \times 87.3 \mathrm{GHz} \cdot \mathrm{mV}^{-1}$.

Since we are in the regime $\Gamma_{N} \gg \Gamma_{S}$, we could expect that, for each dot orbital considered in Sec. III, a fit of

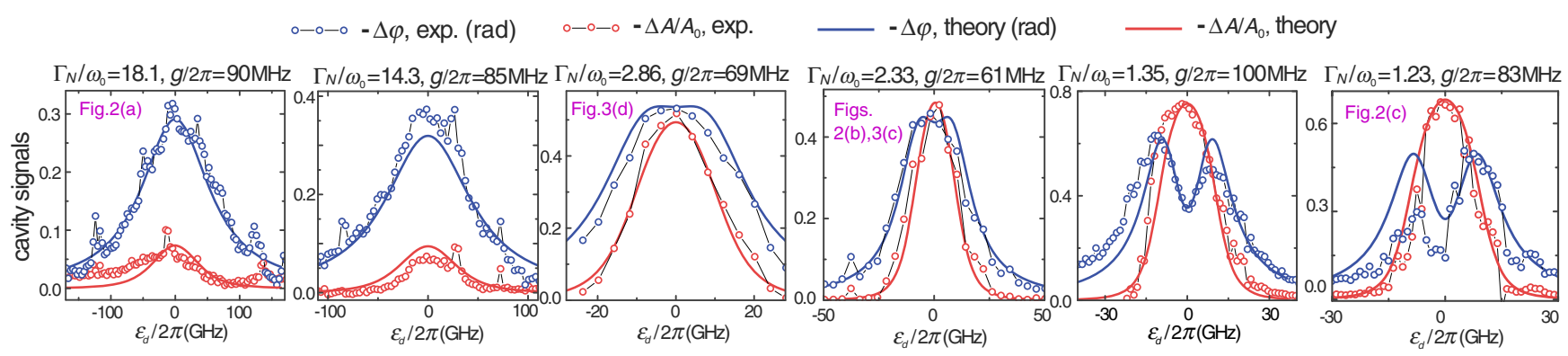

$\Gamma_{N} / \omega_{0}=0.57, g / 2 \pi=66 \mathrm{MHz} \quad \Gamma_{N} / \omega_{0}=0.45, g / 2 \pi=68 \mathrm{MHz} \quad \Gamma_{N} / \omega_{0}=0.38, g / 2 \pi=70 \mathrm{MHz} \quad \Gamma_{N} / \omega_{0}=0.24, g / 2 \pi=120 \mathrm{MHz} \Gamma_{N} / \omega_{0}=0.16, g / 2 \pi=55 \mathrm{MHz} \Gamma_{N} / \omega_{0}=0.14, g / 2 \pi=60 \mathrm{MHz}$
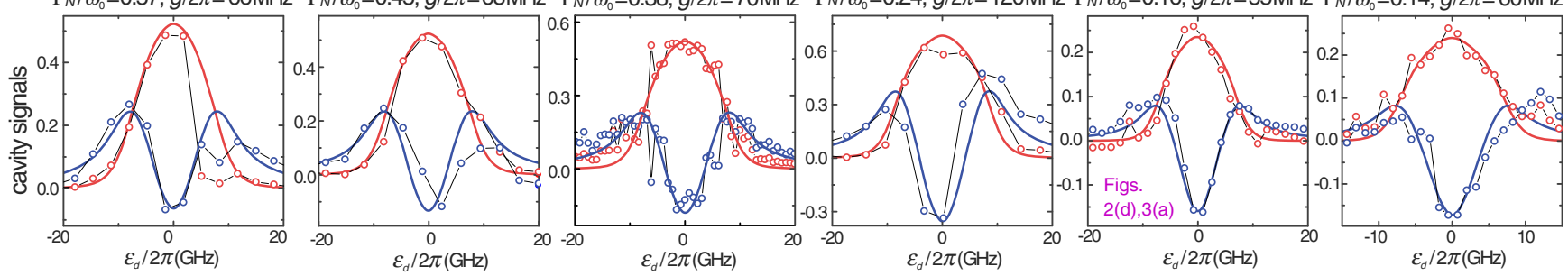

FIG. 7. Cavity signals $\Delta \varphi$ (blue dots) and $\Delta A / A_{0}$ (red dots) versus $\varepsilon_{d}$ for $V_{b}=0$ and different dot orbitals with decreasing tunnel rates $\Gamma_{N}$ from top to bottom and left to right panels. The red and blue lines show the predictions given by Eqs. (5) and (6) for the values of $\Gamma_{N}$ and $g$ given in the different panels and $T=60 \mathrm{mK}$. When a dot-reservoir resonance is already shown in the main text, we indicate the corresponding figure number in pink. 


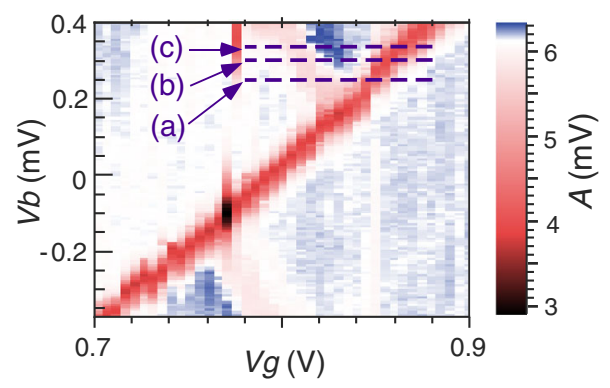

(a) $V_{b}=0.250 \mathrm{mV}$ (b) $V_{b}=0.303 \mathrm{mV}$ (c) $V_{b}=0.336 \mathrm{mV}$

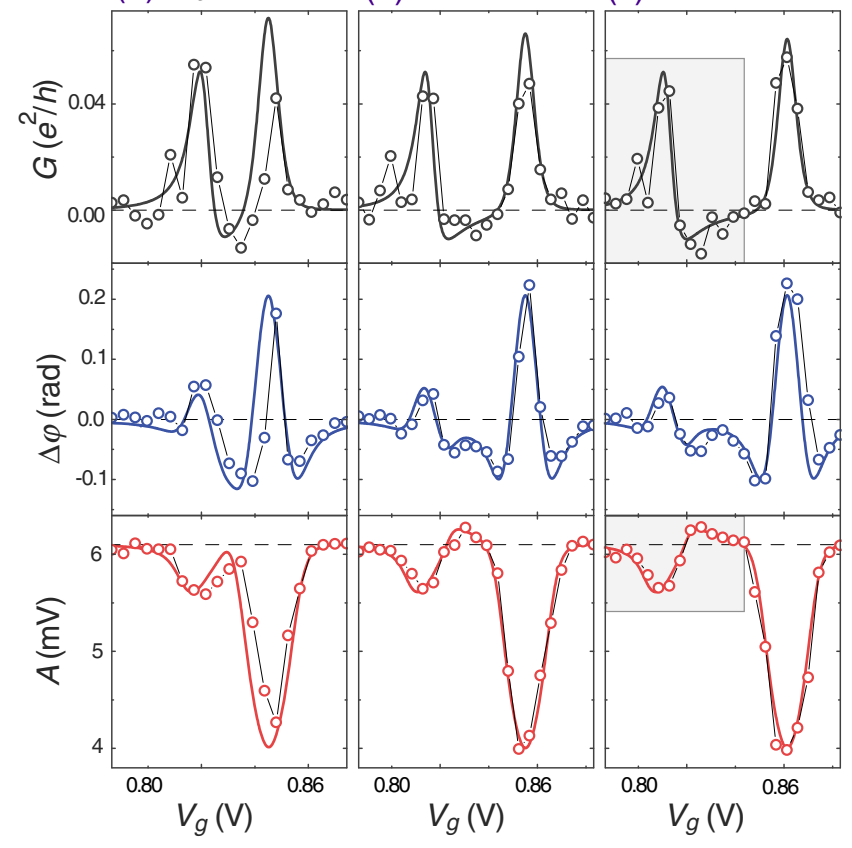

FIG. 8. Top panel: Measured amplitude $A$ versus $V_{b}$ and $V_{g}$, already shown in Fig. 4(c). Bottom panels: Measured conductance $G$ (black dots), and cavity signals $\Delta \varphi$ (blue dots) and $A$ (red dots) versus $V_{g}$, along the dashed lines in the top panel, for $V_{b}=0.25,0.303$, and $0.336 \mathrm{mV}$ from left to right. The solid red, blue, and black lines show the predictions given by Eqs. (3), (6), and (B17), for the same parameters as in Fig. 4. The areas in the gray rectangles are enlarged in Fig. 5 of the main text.

the N-dot conductance peak for a bias voltage $\tilde{V}_{b}>\Delta / e$ should give the value of the $\mathrm{N}$-dot tunnel rate. Then, the only remaining fitting parameter for $\Delta \varphi$ and $\Delta A$ should be $g$. Indeed, for the 6 upper resonances in Fig. 7, a Lorentzian fit of the N-dot conductance peak gives an estimate $\widetilde{\Gamma_{N}}$ of the $\mathrm{N}$-dot tunnel rate, which is in rather good agreement with the value $\Gamma_{N}$ estimated from the cavity signals (see values in Table I). For the highest tunnel rates $\Gamma_{N} / \omega_{0}=$ 18.1 and $\Gamma_{N} / \omega_{0}=14.3$, inaccuracies in the estimation of $\tilde{\Gamma_{N}}$ stem from cotunneling peaks that appear between the Coulomb diamonds and would require a more complete theory. The conductance data for intermediate tunnel rates $1.23<\Gamma_{N} / \omega_{0}<2.86$ are more affected by experimental noise. One can try to minimize these two difficulties by estimating $\widetilde{\Gamma_{N}}$ for values $\tilde{V}_{b}$ of the bias voltage such that cotunneling and experimental noise are reduced. However, for smaller tunnel rates comparable to the temperature, the straightforward estimation of the N-dot tunnel rate from $G$ is not possible anymore because of temperature broadening effects. Therefore, in Sec. III, we preferred to treat $\Gamma_{N}$ as a fitting parameter for the cavity response, knowing that since we have to simultaneously fit two 1D curves with two parameters $\Gamma_{N}$ and $g$, these parameters are strongly constrained anyway.

\section{APPENDIX B: THEORETICAL APPROACH}

\section{Hamiltonian of the quantum dot circuit}

To model the behavior of our setup, we use the total Hamiltonian (1) of the main text, with

$$
\begin{aligned}
H_{d}= & \sum_{\sigma} \varepsilon_{d} \hat{d}_{\sigma}^{\dagger} \hat{d}_{\sigma}+\Delta \sum_{k}\left(\hat{c}_{k \uparrow}^{S_{\uparrow}^{\dagger}} \hat{c}_{-k \downarrow}^{S^{\dagger}}+\text { H.c. }\right) \\
& +\sum_{O \in\{S, N\}, k, \sigma}\left(\varepsilon_{k}^{O} \hat{c}_{k \sigma}^{O \dagger} \hat{c}_{k \sigma}^{O}+\left(t_{O} \hat{d}_{\sigma}^{\dagger} \hat{c}_{k \sigma}^{O}+\text { H.c. }\right)\right) \\
& +\sum_{k, k^{\prime}, \sigma}\left(\varepsilon_{k k^{\prime}}^{n} \hat{b}_{k k^{\prime} \sigma}^{n \dagger} \hat{b}_{k k^{\prime} \sigma}^{n}+\left(t_{n} \hat{b}_{k k^{\prime} \sigma}^{n \dagger} \hat{c}_{k \sigma}^{S}+\text { H.c. }\right)\right),
\end{aligned}
$$

the Hamiltonian of a single quantum dot contacted to a $\mathrm{N}$ and a $\mathrm{S}$ contact. Above $\hat{d}_{\sigma}^{\dagger}\left[\hat{c}_{k \sigma}^{O^{\dagger}}\right]$ is the creation operator for an electron with spin $\sigma$ in the orbital with energy $\epsilon_{d}\left[\epsilon_{k}^{O}\right]$ of the dot [reservoir $O \in\{S, N\}]$. To account for the broadening of the BCS peaks in the density of states of S, we use an auxiliary reservoir $n$ whose states can be populated by the operators $\hat{b}_{k k^{\prime} \sigma}^{n \dagger}$. For simplicity, each level $k \sigma$ of $S$ is coupled to an independent set $k k^{\prime} \sigma$ of levels in $n$. We assume that a bias voltage $V_{b}$ is applied to the $\mathrm{N}$ contact, whereas the $\mathrm{S}$ contact is grounded. For simplicity, we disregard Coulomb interactions in this appendix. Throughout this paper, we use $\hbar=1$ and define the quantities $\Gamma_{N}, \Gamma_{S}, \Gamma_{n}, \varepsilon_{d}, \omega_{0}, \omega_{\mathrm{rf}}, \Delta \omega_{0}, \Delta \Lambda_{0}$, and $g$ as pulsations.

\section{Calculation of the cavity microwave transmission}

The two-port transmission of the cavity can be calculated with the input-output formalism for microwave cavities [90]. In this framework, the bosonic modes $q$ in Eq. (1) include propagating modes in the $L$ and $R$ ports of the cavity, and extra modes accounting for internal cavity damping. The $L$ and $R$ ports cause contributions $\Lambda_{L(R)}$ to the bare cavity linewidth $\Lambda_{0}$, related to the $f_{Q}$ coupling factors and the mode density (see Ref. [90] for details). One can explicitly treat the excitation with frequency $\omega_{\text {rf }}$ imposed on the cavity through the incoming mode $Q$ of the $L$ port $\left(\omega_{\mathrm{rf}}=\omega_{Q}\right)$, by adding to the Hamiltonian (1) the contribution

$$
H_{\mathrm{rf}}=-i\left(f_{Q} \hat{a}^{\dagger} B_{Q} e^{-i \omega_{\mathrm{rf}}\left(t-t_{0}\right)}-f_{Q}^{*} \hat{a} B_{Q}^{*} e^{i \omega_{\mathrm{rf}}\left(t-t_{0}\right)}\right),
$$


with $t_{0}<t$ an initial time before the interaction of the propagating modes with the cavity. The term $H_{\mathrm{rf}}$ corresponds to a classical input signal

$$
b_{i n}=B_{Q} f_{Q} e^{-i \omega_{\mathrm{rf}}\left(t-t_{0}\right)} / \sqrt{2 \Lambda_{L}}
$$

in port $L$. Disregarding quantum fluctuations in the input modes of the cavity, Eqs. (1) and (B2) lead to

$$
\frac{d}{d t} \hat{a}=-i \omega_{0} \hat{a}-i g \hat{n}-\Lambda_{0} \hat{a}-\sqrt{2 \Lambda_{L}} b_{i n},
$$

with $\hat{n}(t)=\sum_{\sigma} \hat{d}_{\sigma}^{\dagger} \hat{d}_{\sigma}$, while the cavity output signal is written as

$$
\hat{b}_{t}=\sqrt{2 \Lambda_{R}} \hat{a} .
$$

If the number of photons in the cavity is larger than about 10 , we can use the semiclassical approximation $\hat{a} \simeq\langle\hat{a}\rangle$. In the linear response limit and stationary regime, $\langle\hat{a}\rangle$ has a negligible component in $e^{i \omega_{\mathrm{rf}} t}$, provided the loaded quality factor of the cavity remains good and $\omega_{\mathrm{rf}} \sim \omega_{0}$. In the framework of Eq. (B1), one can thus estimate the time variations of the average number of electrons in the dot from the linear response to $\hat{a} \simeq \bar{a} e^{-i \omega_{\mathrm{rf}} t}$, as

$$
\langle\hat{n}\rangle(t)=g \tilde{\chi}\left(\omega_{\mathrm{rf}}\right) \bar{a} e^{-i \omega_{\mathrm{rf}} t}+g \tilde{\chi}\left(-\omega_{\mathrm{rf}}\right) \bar{a}^{*} e^{i \omega_{\mathrm{rf}} t} .
$$

This expression involves the dot charge susceptibility

$$
\tilde{\chi}(t)=-i \theta(t)\langle\{\hat{n}(t), \hat{n}(t=0)\}\rangle_{g=0}
$$

calculated in the absence of the cavity. Throughout this appendix, we use the quantum mechanics convention for the Fourier transform, i.e., $\tilde{\chi}(\omega)=\int_{-\infty}^{+\infty} d t \tilde{\chi}(t) e^{i \omega t}$. Injecting Eq. (B6) into the statistical average of Eq. (B4) and disregarding nonresonant terms, we obtain

$$
\bar{a}=\frac{-i f_{Q} B_{Q} e^{i \omega_{\mathrm{rf}} t_{0}}}{\hbar \omega_{\mathrm{rf}}-\hbar \omega_{0}+i \Lambda_{0}-g^{2} \tilde{\chi}\left(\omega_{0}\right)} .
$$

For an agreement with the experimental data, one has to keep in mind that microwave equipment uses the electrical engineering Fourier transform convention, which is complex conjugated to the usual quantum mechanics convention. Hence, combining Eqs. (B3), (B5), and (B8) with $b_{t}=\left\langle\hat{b}_{t}\right\rangle$ and making the substitution $i \rightarrow-i$, we obtain Eq. (2) of the main text, with $\chi(\omega)=\tilde{\chi}(\omega)^{*}$. Note that Refs. [5,34-36] presented related linear response approaches to express the cavity behavior in terms of the charge susceptibility of the quantum dot.

\section{Keldysh description of the quantum dot circuit}

Using the time-dependent Keldysh formalism [91], we obtain the expression (3) of the main text for the dot charge susceptibility. Interestingly, Refs. [25] and [35] introduced related expressions, restricted to the $\mathrm{N}$-dot and Andreev molecular cases, respectively. Equation (3) involves the retarded, advanced, and lesser Green's functions $\breve{\mathcal{G}}^{c}$ of the quantum dot, with $c=r, a$, and $<$, respectively, which have the structure

$$
\check{\mathcal{G}}^{c}=\left[\begin{array}{ll}
\mathcal{G}_{\hat{d}_{\uparrow}, \hat{d}_{\uparrow}^{\dagger}}^{c} & \mathcal{G}_{\hat{d}_{\uparrow}, \hat{d}_{\downarrow}}^{c} \\
\mathcal{G}_{\hat{d}_{\downarrow}^{\dagger}, \hat{d}_{\uparrow}^{\dagger}}^{c} & \mathcal{G}_{\hat{d}_{\downarrow}^{\dagger}, \hat{d}_{\downarrow}^{c}}^{c}
\end{array}\right]
$$

in Nambu space. For any operators $A$ and $B$, we use $\mathcal{G}_{A, B}^{r}(t)=-i \theta(t)\langle\{A(t), B(t=0)\}\rangle$ and $\mathcal{G}_{A, B}^{<}(t)=$ $i\langle B(t=0) A(t)\rangle$. From Hamiltonian (B1), one obtains $[52,92]$

$$
\begin{gathered}
\check{\mathcal{G}}^{r}(\omega)=\left(\check{\mathcal{G}}^{a}(\omega)\right)^{\dagger}=\left[\omega \check{1}-\check{E}_{\mathrm{dot}}-\check{\Sigma}^{r}(\omega)\right]^{-1}, \\
\check{\mathcal{G}}^{<}(\omega)=\check{\mathcal{G}}^{r}(\omega) \check{\Sigma}^{<}(\omega) \check{\mathcal{G}}^{a}(\omega),
\end{gathered}
$$

with

$$
\begin{gathered}
\check{\Sigma}^{r}(\omega)=-i\left(\Gamma_{N} / 2\right) \check{1}-i\left(\Gamma_{S} / 2\right) \check{\mathcal{C}}(\omega), \\
\check{\Sigma}^{<}(\omega)=i \Gamma_{N} \check{f}_{N}(\omega)+i \Gamma_{S} f(\omega) \operatorname{Re}[\check{\mathcal{C}}(\omega)] .
\end{gathered}
$$

Above, we have introduced the diagonal matrices $\breve{1}=\operatorname{diag}(1,1), \quad \breve{E}_{\text {dot }}=\operatorname{diag}\left(\varepsilon_{d},-\varepsilon_{d}\right), \quad$ and $\breve{f}_{N}(\omega)=$ $\operatorname{diag}\left(f\left(\omega-e V_{b}\right), f\left(\omega+e V_{b}\right)\right)$. The terms $\check{\Sigma}^{c}(\omega)$, with $c \in\{r, a,<\}$, describe the effect of the $N$ and $S$ reservoirs on the quantum dot Green's functions in the large bandwidth approximation. We use tunnel rates $\Gamma_{r}=2 \pi\left|t_{r}\right|^{2} \rho_{r}$, with $\rho_{r}$ the density of states per spin direction in reservoir $r \in\{S, N, n\}$. For describing electronic correlations in the superconducting reservoir, we use

$$
\check{\mathcal{C}}(\omega)=\left[\begin{array}{ll}
G_{\omega} & F_{\omega} \\
F_{\omega} & G_{\omega}
\end{array}\right],
$$

with $G_{\omega}=-i\left[\omega+i\left(\Gamma_{n} / 2\right)\right] / D_{\omega}, F_{\omega}=i \Delta / D_{\omega}$, and

$$
D_{\omega}=\sqrt{\Delta^{2}-\left(\omega+i \frac{\Gamma_{n}}{2}\right)^{2}} .
$$

The parameter $\Gamma_{n}$ is often omitted (see, for instance, Refs. [50-53,56,92,93]), but it is essential to account for the broadening of the BCS peaks that is observed experimentally.

In the case $V_{b}=0$ and $\Gamma_{S} \ll \Gamma_{N}, \Delta$, the effect of the superconducting contact can be disregarded, i.e., $\Gamma_{S}=0$. In this limit, Eq. (3) leads to 


$$
\chi(\omega)=\frac{\Gamma_{N}}{\pi \omega\left(i \Gamma_{N}-\omega\right)} \log \left[\frac{4 \epsilon_{d}^{2}+\Gamma_{N}^{2}}{4 \epsilon_{d}^{2}-\left(2 \omega-i \Gamma_{N}\right)^{2}}\right]
$$

for $T=0$ and Eq. (5) for finite $T$. In the case $\Gamma_{S} \neq 0$, we evaluate $\chi(\omega)$ numerically from Eqs. (3), (4), and (B9)-(B15).

For completeness, we mention that the dc current through the spin-degenerate quantum dot can be calculated for $g=0$ as [92]

$$
\begin{aligned}
I= & \frac{2 e \Gamma_{N} \Gamma_{S}}{h} \int d \omega\left(f\left(\omega-e V_{b}\right)-f(\omega)\right)\left[\check{\mathcal{G}}^{r} \operatorname{Re}[\breve{\mathcal{C}}] \check{\mathcal{G}}^{a}\right]_{11} \\
& +\frac{2 e \Gamma_{N}^{2}}{h} \int d \omega\left(f\left(\omega-e V_{b}\right)-f\left(\omega+e V_{b}\right)\right)\left|\check{\mathcal{G}}_{12}^{r}\right|^{2} .
\end{aligned}
$$

This expression includes quasiparticle tunneling as well as Andreev processes. With our noninteracting approach, when $\Gamma_{S}$ increases, subgap Andreev processes appear much more quickly than what is expected in Coulomb blockade regime because Coulomb interactions forbid $2 e$ charge fluctuations necessary for Andreev reflections [93]. In our case, this is not a problem because we have a low $\Gamma_{S}$. In Fig. 4(b), the onset of the noninteracting Andreev current is slightly visible, but this current is barely above the noise level of the data in the top-left panel. For values of $\Gamma_{S}$ larger than in our experiment, it would be necessary to use an interacting theory to satisfactorily reproduce the data.

\section{Keldysh description of the multisite case}

One can generalize the approach of Appendixes B 2 and B 3 to geometries with several quantum dots or sites or several orbitals, denoted with an index $i$. In the case where each discrete level $i \in[1, N]$ of the nanocircuit is shifted by the cavity field $\hat{a}+\hat{a}^{\dagger}$ with a constant $g_{i}$, a semiclassical linear-response description leads to Eqs. (10), (11), and (12) of the main text. These equations involve generalized advanced and retarded Green's functions $\breve{\mathcal{G}}^{a / r}(\omega)$, which enclose $N \times N$ site/orbital sub-blocks. The element $\breve{\mathcal{G}}_{i j}^{a / r}(\omega)$ has a Nambu structure:

$$
\check{\mathcal{G}}_{i j}^{a / r}=\left[\begin{array}{cc}
\mathcal{G}_{\hat{d}_{i \uparrow} / r}^{a / \hat{d}_{j \uparrow}^{\dagger}} & \mathcal{G}_{\hat{d}_{i \uparrow}, \hat{d}_{j \downarrow}}^{a / r} \\
\mathcal{G}_{\hat{d}_{i \downarrow}^{\dagger}, \hat{d}_{j \uparrow}^{\dagger}}^{a} & \mathcal{G}_{\hat{d}_{i \downarrow}^{\dagger}, \hat{d}_{j \downarrow}^{c}}^{c}
\end{array}\right],
$$

with $\mathcal{G}_{A, B}^{a / r}$ scalar Green's functions defined in Appendix B, and $\check{\Sigma}^{<}$the lesser self-energy of the discrete levels. Above $\hat{d}_{i \sigma}^{\dagger}$ is the creation operator for an electron with spin $\sigma$ in the orbital level $i$. The matrix $\check{\tau}_{i}$ is a diagonal matrix that corresponds to $\check{\tau}$ in the orbital block $(i, i)$ and is zero otherwise.
Note that our formalism assumes that the cavity electric field only shifts the discrete energy levels $i$. This can be obtained by using ac top gates to reinforce the coupling between cavity photons and the quantum dot. If one uses a different fabrication technology with, e.g., remote ac gates, it may be necessary to assume that the cavity field also shifts, by a different amount, the potentials of the different reservoirs coupled to the dot. In such a case, one can obtain supplementary effects such as a direct influence of the quantum dot circuit conductance on the cavity linewidth shift $[3,33,36,94]$. A modulation of tunnel couplings by the photonic fields could also be relevant for very high tunnel rates [5]. These cases are beyond the scope of the present article.

[1] A. Wallraff, D. I. Schuster, A. Blais, L. Frunzio, R.-S. Huang, J. Majer, S. Kumar, S. M. Girvin, and R. J. Schoelkopf, Strong Coupling of a Single Photon to a Superconducting Qubit Using Circuit Quantum Electrodynamics, Nature (London) 431, 162 (2004).

[2] L. Childress, A. S. Sørensen, and M. D. Lukin, Mesoscopic Cavity Quantum Electrodynamics with Quantum Dots, Phys. Rev. A 69, 042302 (2004).

[3] M. R. Delbecq, V. Schmitt, F. D. Parmentier, N. Roch, J. J. Viennot, G. Fève, B. Huard, C. Mora, A. Cottet, and T. Kontos, Coupling a Quantum Dot, Fermionic Leads, and a Microwave Cavity on a Chip, Phys. Rev. Lett. 107, 256804 (2011).

[4] T. Frey, P. J. Leek, M. Beck, A. Blais, T. Ihn, K. Ensslin, and A. Wallraff, Dipole Coupling of a Double Quantum Dot to a Microwave Resonator, Phys. Rev. Lett. 108, 046807 (2012).

[5] A. Cottet, T. Kontos, and B. Douçot, Electron-Photon Coupling in Mesoscopic Quantum Electrodynamics, Phys. Rev. B 91, 205417 (2015).

[6] K. D. Petersson, L. W. McFaul, M. D. Schroer, M. Jung, J. M. Taylor, A. A. Houck, and J.R. Petta, Circuit Quantum Electrodynamics with a Spin Qubit, Nature (London) 490, 380 (2012).

[7] M. D. Schroer, M. Jung, K. D. Petersson, and J. R. Petta, Radio Frequency Charge Parity Meter, Phys. Rev. Lett. 109, 166804 (2012).

[8] T. Frey, P. J. Leek, M. Beck, J. Faist, A. Wallraff, K. Ensslin, T. Ihn, and M. Büttiker, Quantum Dot Admittance Probed at Microwave Frequencies with an On-Chip Resonator, Phys. Rev. B 86, 115303 (2012).

[9] H. Toida, T. Nakajima, and S. Komiyama, Vacuum Rabi Splitting in a Semiconductor Circuit QED System, Phys. Rev. Lett. 110, 066802 (2013).

[10] J. Basset, D.-D. Jarausch, A. Stockklauser, T. Frey, C. Reichl, W. Wegscheider, T. M. Ihn, K. Ensslin, and A. Wallraff, Single-Electron Double Quantum Dot DipoleCoupled to a Single Photonic Mode, Phys. Rev. B 88, 125312 (2013).

[11] M.-L. Zhang, D. Wei, G.-W. Deng, S.-X. Li, H.-O. Li, G. Cao, T. Tu, M. Xiao, G.-C. Guo, H.-W. Jiang, and G.-P. Guo, Measuring the Complex Admittance of a Nearly 
Isolated Graphene Quantum Dot, Appl. Phys. Lett. 105, 073510 (2014).

[12] J. J. Viennot, M. R. Delbecq, M. C. Dartiailh, A. Cottet, and T. Kontos, Out-of-Equilibrium Charge Dynamics in a Hybrid Circuit Quantum Electrodynamics Architecture, Phys. Rev. B 89, 165404 (2014).

[13] Y. Y. Liu, K. D. Petersson, J. Stehlik, J. M. Taylor, and J. R. Petta, Photon Emission from a Cavity-Coupled Double Quantum Dot, Phys. Rev. Lett. 113, 036801 (2014).

[14] Y.-Y. Liu, J. Stehlik, C. Eichler, M. J. Gullans, J. M. Taylor, and J.R. Petta, Semiconductor Double Quantum Dot Micromaser, Science 347, 285 (2015).

[15] A. Stockklauser, V. F. Maisi, J. Basset, K. Cujia, C. Reichl, W. Wegscheider, T. Ihn, A. Wallraff, and K. Ensslin, Microwave Emission from Hybridized States in a Semiconductor Charge Qubit, Phys. Rev. Lett. 115, 046802 (2015).

[16] Y.-Y. Liu, J. Stehlik, M. J. Gullans, J. M. Taylor, and J. R. Petta, Injection Locking of a Semiconductor Double Quantum Dot Micromaser, Phys. Rev. A 92, 053802 (2015).

[17] J. J. Viennot, M. C. Dartiailh, A. Cottet, and T. Kontos, Coherent Coupling of a Single Spin to Microwave Cavity Photons, Science 349, 408 (2015).

[18] A. Cottet, T. Kontos, S. Sahoo, H. T. Man, M.-S. Choi, W. Belzig, C. Bruder, A. F. Morpurgo, and C. Schönenberger, Nanospintronics with Carbon Nanotubes, Semicond. Sci. Technol. 21, S78 (2006).

[19] L. Hofstetter, S. Csonka, J. Nygard, and C. Schönenberger, Cooper Pair Splitter Realized in a Two-Quantum-Dot Y-Junction, Nature (London) 461, 960 (2009).

[20] L. G. Herrmann, F. Portier, P. Roche, A. L. Yeyati, T. Kontos, and C. Strunk, Carbon Nanotubes as Cooper-Pair Beam Splitters, Phys. Rev. Lett. 104, 026801 (2010).

[21] J.-D. Pillet, C. Quay, P. Morfin, C. Bena, A. Levy Yeyati, and P. Joyez, Andreev Bound States in SupercurrentCarrying Carbon Nanotubes Revealed, Nat. Phys. 6, 965 (2010).

[22] T. Dirks, T. L. Hughes, S. Lal, B. Uchoa, Y.-F. Chen, C. Chialvo, P. M. Goldbart, and N. Mason, Transport through Andreev Bound States in a Graphene Quantum Dot, Nat. Phys. 7, 386 (2011).

[23] C. Janvier, L. Tosi, L. Bretheau, Ç. Ö. Girit, M. Stern, P. Bertet, P. Joyez, D. Vion, D. Esteve, M. F. Goffman, H. Pothier, and C. Urbina, Coherent Manipulation of Andreev States in Superconducting Atomic Contacts, Science 349, 1199 (2015).

[24] V. Mourik, K. Zuo, S. M. Frolov, S. R. Plissard, E. P. A. M. Bakkers, and L. P. Kouwenhoven, Signatures of Majorana Fermions in Hybrid Superconductor-Semiconductor Nanowire Devices, Science 336, 1003 (2012).

[25] J. Sköldberg, T. Löfwander, V.S. Shumeiko, and M. Fogelström, Spectrum of Andreev Bound States in a Molecule Embedded Inside a Microwave-Excited Superconducting Junction, Phys. Rev. Lett. 101, 087002 (2008).

[26] A. Cottet, T. Kontos, and A. Levy Yeyati, Subradiant Split Cooper Pairs, Phys. Rev. Lett. 108, 166803 (2012).

[27] A. Cottet, Probing Coherent Cooper Pair Splitting with Cavity Photons, Phys. Rev. B 90, 125139 (2014).

[28] M. Trif and Y. Tserkovnyak, Resonantly Tunable Majorana Polariton in a Microwave Cavity, Phys. Rev. Lett. 109, 257002 (2012).
[29] T. L. Schmidt, A. Nunnenkamp, and C. Bruder, Majorana Qubit Rotations in Microwave Cavities, Phys. Rev. Lett. 110, 107006 (2013).

[30] A. Cottet, T. Kontos, and B. Douçot, Squeezing Light with Majorana Fermions, Phys. Rev. B 88, 195415 (2013).

[31] O. Dmytruk, M. Trif, and P. Simon, Cavity Quantum Electrodynamics with Mesoscopic Topological Superconductors, Phys. Rev. B 92, 245432 (2015).

[32] R. Chirla, A. Manolescu, and C. P. Moca, Transmission of a Microwave Cavity Coupled to Localized Shiba States, Phys. Rev. B 93, 155110 (2016).

[33] M. R. Delbecq, L. E. Bruhat, J. J. Viennot, S. Datta, A. Cottet, and T. Kontos, Photon-Mediated Interaction between Distant Quantum Dot Circuits, Nat. Commun. 4, 1400 (2013).

[34] A. Cottet, C. Mora, and T. Kontos, Mesoscopic Admittance of a Double Quantum Dot, Phys. Rev. B 83, 121311(R) (2011).

[35] M. Schiró and K. Le Hur, Tunable Hybrid Quantum Electrodynamics from Nonlinear Electron Transport, Phys. Rev. B 89, 195127 (2014).

[36] O. Dmytruk, M. Trif, C. Mora, and P. Simon, Cavity Quantum Electrodynamics with an Out-of-Equilibrium Quantum Dot, Phys. Rev. B 93, 075425 (2016).

[37] M. Büttiker, H. Thomas, and A. Prêtre, Mesoscopic Capacitors, Phys. Lett. A 180, 364 (1993).

[38] A. Prêtre, H. Thomas, and M. Büttiker, Dynamic Admittance of Mesoscopic Conductors: Discrete-Potential Model, Phys. Rev. B 54, 8130 (1996).

[39] J. Gabelli, G. Fève, J.-M. Berroir, B. Plaçais, A. Cavanna, B. Etienne, Y. Jin, and D. C. Glattli, Violation of Kirchhoff's Laws for a Coherent RC Circuit, Science 313, 499 (2006).

[40] G. Fève, A. Mahé, J.-M. Berroir, T. Kontos, B. Plaçais, C. Glattli, A. Cavanna, B. Etienne, and Y. Jin, An On-Demand Coherent Single Electron Source, Science 316, 1169 (2007).

[41] S. Nigg, R. Lopez, and M. Büttiker, Mesoscopic Charge Relaxation, Phys. Rev. Lett. 97, 206804 (2006).

[42] Ya. I. Rodionov, I. S. Burmistrov, and A. S. Ioselevich, Charge Relaxation Resistance in the Coulomb Blockade Problem, Phys. Rev. B 80, 035332 (2009).

[43] C. Mora and K. Le Hur, Universal Resistances of the Quantum RC Circuit, Nat. Phys. 6, 697 (2010).

[44] Y. Hamamoto, T. Jonckheere, T. Kato, and T. Martin, Dynamic Response of a Mesoscopic Capacitor in the Presence of Strong Electron Interactions, Phys. Rev. B 81, 153305 (2010).

[45] M. Filippone, K. Le Hur, and C. Mora, Giant Charge Relaxation Resistance in the Anderson Model, Phys. Rev. Lett. 107, 176601 (2011).

[46] M. Lee, R. Lopez, M.-S. Choi, T. Jonckheere, and T. Martin, Many-Body Correlation Effect on Mesoscopic Charge Relaxation, Phys. Rev. B 83, 201304 (2011).

[47] I. S. Burmistrov and Ya. I. Rodionov, Charge Relaxation Resistance in the Cotunneling Regime of Multi-Channel Coulomb Blockade: Violation of Korringa-Shiba Relation, Phys. Rev. B 92, 195412 (2015).

[48] T. W. Larsen, K. D. Petersson, F. Kuemmeth, T. S. Jespersen, P. Krogstrup, J. Nygard, and C. M. Marcus, A Semiconductor Nanowire-Based Superconducting Qubit, Phys. Rev. Lett. 115, 127001 (2015). 
[49] G. de Lange, B. van Heck, A. Bruno, D. J. van Woerkom, A. Geresdi, S. R. Plissard, E.P. A. M. Bakkers, A. R. Akhmerov, and L. DiCarlo, Realization of Microwave Quantum Circuits Using Hybrid SuperconductingSemiconducting Nanowire Josephson Elements, Phys. Rev. Lett. 115, 127002 (2015).

[50] C. B. Whan and T. P. Orlando, Transport Properties of a Quantum Dot with Superconducting Leads, Phys. Rev. B 54, R5255(R) (1996).

[51] H.-K. Zhao and G.v. Gehlen, Mesoscopic Transport through a Normal-Metal-Quantum-Dot-Superconductor System with ac Responses, Phys. Rev. B 58, 13660 (1998).

[52] Q.-f. Sun, J. Wang, and T.-h. Lin, Photon-Assisted Andreev Tunneling through a Mesoscopic Hybrid System, Phys. Rev. B 59, 13126 (1999).

[53] S. Y. Cho, K. Kang, and C.-M. Ryu, Photon-Assisted Quasiparticle Transport and Andreev Transport through an Interacting Quantum Dot, Phys. Rev. B 60, 16874 (1999).

[54] Y. Zhu, W. Li, T.-h. Lin, and Q.-f. Sun, Microwave-Induced $\pi$-Junction Transition in a Superconductor/Quantum Dot/Superconductor Structure, Phys. Rev. B 66, 134507 (2002).

[55] T. K. T. Nguyen, T. Jonckheere, A. Crépieux, A. V. Nguyen, and T. Martin, Photoassisted Andreev Reflection as a Probe of Quantum Noise, Phys. Rev. B 76, 035421 (2007).

[56] A. Nurbawono, Y. P. Feng, and C. Zhang, Electron Tunneling through a Hybrid Superconducting-Normal Mesoscopic Junction under Microwave Radiation, Phys. Rev. B 82, 014535 (2010).

[57] A. G. Moghaddam, M. Governale, and J. König, Driven Superconducting Proximity Effect in Interacting Quantum Dots, Phys. Rev. B 85, 094518 (2012).

[58] R. Avriller and F. Pistolesi, Andreev Bound-State Dynamics in Quantum-Dot Josephson Junctions: A Washing Out of the 0- $\pi$ Transition, Phys. Rev. Lett. 114, 037003 (2015).

[59] J. Baranski and T. Domanski, Enhancements of the Andreev Conductance due to Emission/Absorption of Bosonic Quanta, J. Phys. Condens. Matter 27, 305302 (2015).

[60] If one uses two $\mathrm{N}$ contacts with a similar structure and the same bias voltage, this only increases the effective density of states $\rho_{N}$ of the normal continuum seen by the quantum dot. In this picture, the effective tunnel rate $\Gamma_{N}=2 \pi\left|t_{N}\right|^{2} \rho_{N}$ corresponds to the sum of the tunnel rates of the two real $\mathrm{N}$ contacts.

[61] J. Wang, B. Wang, and H. Guo, Quantum Inductance and Negative Electrochemical Capacitance at Finite Frequency in a Two-Plate Quantum Capacitor, Phys. Rev. B 75, 155336 (2007).

[62] This mapping is valid as long as the cavity resonance is well described by a Lorentzian, which is the case we are concerned with [see Eq. (10)]. In particular, the prefactor $t_{0}$, which characterizes our setup transmission, is assumed to be frequency independent in the explored range, and by definition, it is not changed by the nanocircuit.

[63] In the linear limit where $\Delta \varphi$ and $\Delta A$ are small, one has $\Delta \omega_{0} \simeq \Lambda_{0} \Delta \varphi$ and $\Delta \Lambda_{0} \simeq-\Lambda_{0} \Delta A / A_{0}$ so that $\Delta \omega_{0}$ and $\Delta \Lambda_{0}$ reveal the cavity frequency and linewidth shifts, respectively. However, since we have strong signals, it is more accurate to use the nonlinear relations of the main text.
[64] H. Shiba, The Korringa Relation for the Impurity Nuclear Spin Lattice Relaxation in Dilute Kondo Alloys, Prog. Theor. Phys. 54, 967 (1975).

[65] Y. Meir, N. S. Wingreen, and P. A. Lee, Transport through a Strongly Interacting Electron System: Theory of Periodic Conductance Oscillations, Phys. Rev. Lett. 66, 3048 (1991).

[66] A. Martin-Rodero, A. Levy Yeyati, F. Flores, and R. C. Monreal, Interpolative Approach for Electron and ElectronPhonon Interactions: From the Kondo to the Polaronic Regime, Phys. Rev. B 78, 235112 (2008).

[67] T. Dirks, Y.-F. Chen, N. O. Birge, and N. Mason, Superconducting Tunneling Spectroscopy of a Carbon Nanotube Quantum Dot, Appl. Phys. Lett. 95, 192103 (2009).

[68] S. Pfaller, A. Donarini, and M. Grifoni, Subgap Features Due to Quasiparticle Tunneling in Quantum Dots Coupled to Superconducting Leads, Phys. Rev. B 87, 155439 (2013).

[69] J. Gramich, A. Baumgartner, and C. Schönenberger, Resonant and Inelastic Andreev Tunneling Observed on a Carbon Nanotube Quantum Dot, Phys. Rev. Lett. 115, 216801 (2015).

[70] D. C. Ralph, C. T. Black, M. Tinkham, D. C. Ralph, C. T. Black, and M. Tinkham, Spectroscopic Measurements of Discrete Electronic States in Single Metal Particles, Phys. Rev. Lett. 74, 3241 (1995).

[71] P. K. Tien and J. P. Gordon, Multiphoton Process Observed in the Interaction of Microwave Fields with the Tunneling between Superconductor Films, Phys. Rev. 129, 647 (1963).

[72] L. P. Kouwenhoven, S. Jauhar, J. Orenstein, P. L. McEuen, Y.Nagamune, J. Motohisa, and H. Sakaki, Observation of Photon-Assisted Tunneling through a Quantum Dot, Phys. Rev. Lett. 73, 3443 (1994).

[73] L. P. Kouwenhoven, S. Jauhar, K. McCormick, D.Dixon, P. L. McEuen, Yu. V. Nazarov, N. C. van der Vaart, and C. T. Foxon, Photon-Assisted Tunneling through a Quantum Dot, Phys. Rev. B 50, 2019 (1994).

[74] R. H. Blick, R. J. Haug, D. W. van der Weide, K. von Klitzing, and K. Eberl, Photon-Assisted Tunneling through a Quantum Dot at High Microwave Frequencies, Appl. Phys. Lett. 67, 3924 (1995).

[75] In the semiclassical limit, by combining Eqs. (B4) and (B6) with $\chi\left(-\omega_{0}\right)=\chi\left(\omega_{0}\right)^{*}$, one obtains a photon emission/ absorption rate $\Gamma_{e / a}=-2 g^{2} \operatorname{Im}\left[\chi\left(\omega_{0}\right)\right] n_{p h}$. Then, from Ref. [63], for linear cavity signals, one has $\Delta \Lambda_{0}=$ $g^{2} \operatorname{Im}\left[\chi\left(\omega_{0}\right)\right] \simeq-\Lambda_{0} \Delta A / A_{0}$. This gives $\Gamma_{e / a} \simeq 2 n_{p h} \Lambda_{0} \Delta A / A_{0}$.

[76] S. DeFranceschi, L. Kouwenhoven, C. Schönenberger, and W. Wernsdorfer, Hybrid Superconductor-Quantum Dot Devices, Nat. Nanotechnol. 5, 703 (2010).

[77] C. Feuillet-Palma, T. Delattre, P. Morfin, J.-M. Berroir, G. Fève, D. C. Glattli, B. Plaçais, A. Cottet, and T. Kontos, Conserved Spin and Orbital Phase along Carbon Nanotubes Connected with Multiple Ferromagnetic Contacts, Phys. Rev. B 81, 115414 (2010).

[78] A. D. Crisan, S. Datta, J. J. Viennot, M. R. Delbecq, A. Cottet, and T. Kontos, Harnessing Spin Precession with Dissipation, Nat. Commun. 7, 10451 (2016).

[79] R. Leturcq, D. Graf, T. Ihn, K. Ensslin, D. D. Driscoll, and A. C. Gossard, Multi-Terminal Transport through a Quantum Dot in the Coulomb-Blockade Regime, Europhys. Lett. 67, 439 (2004). 
[80] As a first test, we have checked that the multisite Eq. (11) agrees with the expressions given in Ref. [34] for a noninteracting equilibrium double quantum dot with $\mathrm{N}$ reservoirs.

[81] In the multidot case, we define $\chi_{i j}(t)=-i \theta(t)\left\langle\left\{\hat{n}_{i}(t)\right.\right.$, $\left.\left.\hat{n}_{j}(t=0)\right\}\right\rangle_{0}$, where $\hat{n}_{i}$ is the quantum operator for the number of electrons in site $i$ and \langle\rangle$_{0}$ denotes the statistical averaging with $g_{i}=0$ for $i \in[1, N]$.

[82] For instance, in the case of a noninteracting single quantum dot in equilibrium with $\mathrm{N}$ reservoirs, Eq. (5) can be recovered from the Landauer Büttiker scattering description used in Ref. [8].

[83] P. Trocha and J. Barnaś, Spin-Polarized Andreev Transport Influenced by Coulomb Repulsion through a Two-QuantumDot System, Phys. Rev. B 89, 245418 (2014).

[84] A. Sarlette, J. M. Raimond, M. Brune, and P. Rouchon, Stabilization of Nonclassical States of the Radiation Field in a Cavity by Reservoir Engineering, Phys. Rev. Lett. 107, 010402 (2011).

[85] E. T. Holland, B. Vlastakis, R. W. Heeres, M. J. Reagor, U. Vool, Z. Leghtas, L. Frunzio, G. Kirchmair, M. H. Devoret, M. Mirrahimi, and R. J. Schoelkopf, Single-Photon Resolved Cross-Kerr Interaction for Autonomous Stabilization of Photon-Number States, Phys. Rev. Lett. 115, 180501 (2015).

[86] G. A. Steele, A. K. Hüttel, B. Witkamp, M. Poot, H. B. Meerwaldt, L. P. Kouwenhoven, and H. S. J. van der Zant,
Strong Coupling between Single-Electron Tunneling and Nanomechanical Motion, Science 325, 1103 (2009).

[87] J. J. Viennot, J. Palomo, and T. Kontos, Stamping Single Wall Nanotubes for Circuit Quantum Electrodynamics, Appl. Phys. Lett. 104, 113108 (2014).

[88] L. E. Bruhat et al. (in preparation).

[89] To be published elsewhere.

[90] A. A. Clerk, M. H. Devoret, S. M. Girvin, F. Marquardt, and R. J. Schoelkopf, Introduction to Quantum Noise, Measurement and Amplification, Rev. Mod. Phys. 82, 1155 (2010).

[91] A.-P. Jauho, N. S. Wingreen, and Y. Meir, Time-Dependent Transport in Interacting and Noninteracting ResonantTunneling Systems, Phys. Rev. B 50, 5528 (1994).

[92] Qing-feng Sun, Jian Wang, and Tsung-han Lin, Resonant Andreev Reflection in a Normal-Metal-Quantum-DotSuperconductor System, Phys. Rev. B 59, 3831 (1999).

[93] K. Kang, Multiple Andreev Reflections through an Interacting Quantum Dot, Physica (Amsterdam) 5E, 36 (1999).

[94] N. Ares, F. J. Schupp, A. Mavalankar, G. Rogers, J. Griffiths, G. A. C. Jones, I. Farrer, D. A. Ritchie, C. G. Smith, A. Cottet, G. A. D. Briggs, and E. A. Laird, Sensitive Radio-Frequency Measurements of a Quantum Dot by Tuning to Perfect Impedance Matching, Phys. Rev. Applied 5, 034011 (2016). 